\title{
No evidence of effects or interaction between the widely used herbicide, glyphosate, and a common parasite in bumble bees
}

\author{
Edward A Straw ${ }^{\text {Corresp., } 1}$, Mark J. F. Brown ${ }^{1}$ \\ ${ }^{1}$ Department of Biological Sciences, School for Life Sciences and the Environment, Royal Holloway University of London, Egham, United Kingdom \\ Corresponding Author: Edward A Straw \\ Email address: ed.straw.2018@live.rhul.ac.uk
}

Background. Glyphosate is the world's most used pesticide and it is used without the mitigation measures that could reduce the exposure of pollinators to it. However, studies are starting to suggest negative impacts of this pesticide on bees, an essential group of pollinators. Accordingly, whether glyphosate, alone or alongside other stressors, is detrimental to bee health is a vital question. Bees are suffering declines across the globe, and pesticides, including glyphosate, have been suggested as being factors in these declines.

Methods. Here we test, across a range of experimental paradigms, whether glyphosate impacts a wild bumble bee species, Bombus terrestris. In addition, we build upon existing work with honey bees testing glyphosate-parasite interactions by conducting fully crossed experiments with glyphosate and a common bumble bee trypanosome gut parasite, Crithidia bombi. We utilised regulatory acute toxicity testing protocols, modified to allow for exposure to multiple stressors. These protocols are expanded upon to test for effects on long term survival (20 days). Microcolony testing, using unmated workers, was employed to measure the impacts of either stressor on a proxy of reproductive success. This microcolony testing was conducted with both acute and chronic exposure to cover a range of exposure scenarios.

Results. We found no effects of acute or chronic exposure to glyphosate, over a range of timespans post-exposure, on mortality or a range of sublethal metrics. We also found no interaction between glyphosate and Crithidia bombi in any metric, although there was conflicting evidence of increased parasite intensity after an acute exposure to glyphosate. In contrast to published literature, we found no direct impacts of this parasite on bee health. Our testing focussed on mortality and worker reproduction, so impacts of either or both of these stressors on other sublethal metrics could still exist.

Conclusions. Our results expand the current knowledge on glyphosate by testing a previously untested species, Bombus terrestris, using acute exposure, and by incorporating a parasite never before tested alongside glyphosate. In conclusion our results find that glyphosate, as an active ingredient, is unlikely to be harmful to bumble bees either alone, or alongside Crithidia bombi. 
1 No evidence of effects or interaction between the widely used

2 herbicide, glyphosate, and a common parasite in bumble bees.

Edward A. Straw ${ }^{1}$, Mark J. F. Brown ${ }^{1}$

5

${ }^{1}$ Centre for Ecology, Evolution \& Behaviour, Department of Biological Sciences, School for Life Sciences and the Environment, Royal Holloway University of London, Egham, Surrey, TW20 OEX, UK.

Corresponding Author:

Edward A. Straw ${ }^{1 *}$

Email address: EdwardAStraw@gmail.com

\section{Abstract}

Background. Glyphosate is the world's most used pesticide and it is used without the mitigation measures that could reduce the exposure of pollinators to it. However, studies are starting to suggest negative impacts of this pesticide on bees, an essential group of pollinators. Accordingly, whether glyphosate, alone or alongside other stressors, is detrimental to bee health is a vital question. Bees are suffering declines across the globe, and pesticides, including glyphosate, have been suggested as being factors in these declines.

Methods. Here we test, across a range of experimental paradigms, whether glyphosate impacts a wild bumble bee species, Bombus terrestris. In addition, we build upon existing work with honey bees testing glyphosate-parasite interactions by conducting fully crossed experiments with glyphosate and a common bumble bee trypanosome gut parasite, Crithidia bombi. We utilised regulatory acute toxicity testing protocols, modified to allow for exposure to multiple stressors. These protocols are expanded upon to test for effects on long term survival (20 days). Microcolony testing, using unmated workers, was employed to measure the impacts of either stressor on a proxy of reproductive success. This microcolony testing was conducted with both acute and chronic exposure to cover a range of exposure scenarios.

Results. We found no effects of acute or chronic exposure to glyphosate, over a range of timespans post-exposure, on mortality or a range of sublethal metrics. We also found no interaction between glyphosate and Crithidia bombi in any metric, although there was conflicting evidence of increased parasite intensity after an acute exposure to glyphosate. In contrast to published literature, we found no direct impacts of this parasite on bee health. Our testing focussed on mortality and worker reproduction, so impacts of either or both of these stressors on other sublethal metrics could still exist. untested species, Bombus terrestris, using acute exposure, and by incorporating a parasite 
39

40

41

42

43

44

45

46

47

48

49

50

51

52

53

54

55

56

57

58

59

60

61

62

63

64

65

66

67

68

69

70

71

72

73

74

75

76

active ingredient, is unlikely to be harmful to bumble bees either alone, or alongside Crithidia bombi.

\section{Introduction}

Glyphosate is the world's most used pesticide (Duke and Powles, 2008, Benbrook 2016). It is a herbicide used to suppress weeds in agricultural and amenity settings (Duke and Powles, 2008, Duke, 2018). Glyphosate helps reduce the need for tilling and mechanical weeding, which helps protect against soil erosion and boosts farmers yields and profits (Becki, Flower and Ashworth 2020). Bees are exposed to glyphosate frequently in nature through spraying of weeds, contamination of water, and application onto glyphosate resistant flowering crops (Odemer et al. 2020, Straw, Carpentier and Brown, 2021). Glyphosate-based herbicide products typically do not carry any mitigation measures aimed at reducing bees exposure to them. Research into herbicides, and glyphosate specifically, has grown considerably in recent years, with just 15 papers found in a systematic review of literature up to 2018 (Cullen et al. 2019), the first of which was published in 2011, while five were published in the final year searched. Several more publications have emerged since then (e.g. Odemer et al. 2020, Motta et al. 2020, Motta et al. 2020). To date most of these studies have used honey bees, Apis mellifera, with only a few testing the impacts on other bee species (Abraham et al. 2018, Seide et al. 2018, Ruiz-Toledo and Sánchez-Guillén, 2014). Glyphosate has also undergone regulatory testing for governmental authorities worldwide to determine its effects on bees (EFSA, 2015, Duke 2018). Glyphosate is currently approved in all major territories (Duke 2018), and where it is not approved (Mexico, for example) this is for human health reasons, not bee health reasons (Alcántara-de la Cruz et al. 2021).

In the European Union (EU) and North America pesticide regulation uses a tiered approach, with initial toxicity testing focussing solely on mortality (lower tier), and, if toxicity thresholds are met in the lower tier tests, then more complex experiments are conducted (higher tier) (EFSA, 2013, EPA, 2014). In the EU specifically this initial testing comprises two tests, acute oral exposure and acute contact exposure, both performed with the pure active ingredient and the representative formulation (EFSA, 2013). It has been suggested that this mortality-focussed approach is inadequate to properly assess the toxicity of a substance, and that there should be a move towards a fitness-based approach that also considers sublethal and reproductive effects at lower tiers (Straub, Strobl and Neumann, 2020). In the EU, glyphosate did not meet the toxicity thresholds required to trigger higher tier testing, so was approved for use with only very minimal bee testing (EFSA, 2015). Alongside regulatory testing a number of academic experiments have found that oral exposure to glyphosate does not cause mortality in adult bees (Herbert et al. 2014, Goñalons and Farina 2018, Motta, Raymann and Moran 2019, Blot et al. 2019, Faita et al. 2020, Almasri et al. 2021), although there is mixed evidence, with Almasri 
77 et al. (2020) and Motta and Moran (2020) finding mortality at doses considerably lower than

78

79

80

81

82

83

84

85

86

87

88

89

90

91

92

93

94

95

96

97

98

99

100

101

102

103

104

105

106

107

108

109

110

111

112

113

doses found to be non-lethal in other work.

While there is little strong evidence that glyphosate causes mortality in adult bees, it has been found to cause a range of sublethal effects in honey bees (reviewed in Farina et al. 2019). Chronic exposure to field realistic doses has been found to impair learning (Herbert et al. 2014, Balbuena et al. 2015) and increase the length of time taken to return to a colony (Balbuena et al. 2015). Chronic exposure has also been linked to larval mortality, reduced body mass, and a reduction in successful moulting (Vazquez et al. 2018), although the evidence here is mixed with conflicting results across years and colonies. At a molecular level, glyphosate has been found to impair antioxidant and acetylcholinesterase production (Helmer et al. 2015, Boily et al. 2013). While these results are limited in their scope, and derive only from honey bees, they represent clear evidence that the herbicide glyphosate can be biologically active in bees, and that examining the mortality effects of glyphosate in isolation are insufficient to understand its impacts on bees.

Motta, Raymann and Moran (2018) and Motta et al. (2020) found that in honey bees chronic exposure to glyphosate does not typically cause significant mortality, but that glyphosate can synergise with parasites to cause mortality. Exposure to the opportunistic parasite Serratia marcescens caused some mortality, around $20-30 \%$ more than the control, while glyphosate caused no more mortality than the control. However, when both stressors were applied simultaneously the mortality increased by almost $80 \%$ compared to the control. This result was replicated with a glyphosate-based formulation, Roundup ${ }^{\circledR}$ ProMAX, in Motta et al. (2020), showing that the formulation also causes the synergism. Glyphosate induced a knockdown of protective gut bacteria that allowed the parasite to be more deadly, thus explaining how an otherwise non-lethal pesticide synergises to cause substantial mortality. This result highlights the importance of testing multiple stressors on bees, as even individually non-lethal pesticides can cause considerable synergism alongside common parasites.

In addition to this synergism, there is mixed evidence for the interaction between another bee parasite group, Nosema spp., and glyphosate in honey bees. Both Blot et al. (2019) and Faita et al. (2020) found no effect of chronic exposure to glyphosate on mortality and a significant effect of Nosema spp.. However, only Faita et al. (2020) observed a significant interaction between the two stressors, with a $17 \%$ increase in mortality compared to the Nosema spp. alone. This difference may be attributable to the use of a formulation by Faita et al. (2020), rather than just the active ingredient used by Blot et al. (2020), or the mix of Nosema apis and Nosema ceranae used by Faita et al. (2020), rather than just Nosema ceranae used by Blot et al. (2020). In fact, 
114 the use of a formulation in Faita et al. (2020) does prevent the effect observed being attributable to glyphosate as the other ingredients may have driven the effect.

116

The studies described above focus on honey bees and common parasites. Here we extend this approach to bumble bees (Bombus spp.) and their common trypanosome gut parasite Crithidia bombi, which has been found at prevalences of up to $82 \%$ in the wild (Gillespie 2010), although this level of infection is not found in all studies, with large variation between years, sites and species (Shykoff and Schmid-Hempel 1999, Korner and Schmid-Hempel, 2005, Rutrecht and Brown, 2008, Gillespie 2010, Jones and Brown, 2014, Hicks et al. 2018). C. bombi is likely less damaging of a parasite to Bombus terrestris than either Nosema spp. or S. marcescens are to honey bees, with no individual effect on mortality in otherwise unstressed bees (Brown, Loosli, and Schmid-Hempel 2000, Fauser-Misslin et al. 2014, Baron, Raine and Brown 2014). At the colony level, uncontrolled or post-founding infections have no impact on growth or production of sexuals (Shykoff and Schmid-Hempel, 1991, Fauser-Misslin et al. 2014). In contrast, when experimentally infected bees are starved, worker mortality rates increase by $50 \%$ (Brown, Loosli, and Schmid-Hempel, 2000), and when infections are experimentally controlled and occur before the stressful hibernation period, the parasite has dramatic negative impacts of up to $40 \%$ on host fitness (Brown, Schmid-Hempel, and Schmid-Hempel 2003, Yourth, Brown and Schmid-Hempel, 2008). Thus, this parasite is most likely to have impacts on bumble bees when combined with other stressors.

134

Finally, C. bombi infection is strongly related to the host gut microbiome, with specific bacterial groups like Apibacter, Lactobacillus Firm-5 and Gilliamella conferring increased resistance in $B$. terrestris (Koch and Schmid-Hempel, 2011, Mockler et al. 2018). Interestingly, a range of studies have found an effect of glyphosate on the honey bee microbiome (Dai et al. 2018, Motta, Raymann and Moran, 2018, Motta and Moran, 2020, Blot et al. 2019, Motta et al. 2020), consistently finding that it changes the microbiome composition. This suggests that, despite differences between $A$. mellifera and $B$. terrestris and their microbiomes, glyphosate might impact $C$. bombi indirectly through modifications of the gut microbiome.

In this study, we test whether glyphosate has direct impacts on worker mortality or reproduction, whether it interacts with $C$. bombi to impact these metrics of bee health, and whether infected bumble bees that are exposed to glyphosate, either acutely or chronically, will have increased C. bombi intensities. 


\section{Materials \& Methods}

149

150

151

152

153

154

155

156

157

158

159

160

161

162

163

164

165

166

167

168

169

170

171

172

173

174

175

176

177

178

179

180

181

\section{General}

Bombus terrestris audax colonies were ordered from Agralan Ltd, Swindon, UK. Colonies were maintained on ad libitum sucrose and honey bee collected pollen from Thorne, Windsor, UK and Agralan Ltd, Swindon, UK respectively. On arrival, 10 workers per colony were removed and their faeces screened for micro-parasites (Rutrecht \& Brown, 2008). No infections were detected, and all colonies were thus retained in the experiment. Further molecular screening may have revealed viral presence (Graystock et al. 2013) undetectable by visual screening, but this was beyond the scope of our study. Random allocation of colonies to experiments, and the use of internal colony controls, mitigated against any such infections having impacts on our results. The number of bees or microcolonies included in each treatment group is presented in Supplementary Tables 1-5. Bees were removed from their colonies without regard for their age, hence bee age is not controlled between treatments, however it was randomly distributed between treatments. Pesticides were applied as pure active ingredient, glyphosate (SigmaAldrich) CAS-no: 1071-83-6 and dimethoate (Sigma-Aldrich) CAS-no: 60-51-5.

\section{Modified Ecotoxicological Protocol OECD 247: General Methods}

OECD 247 (OECD, 2017) is an internationally agreed upon protocol for testing the toxicity effects of acute exposure to an oral solution in bumble bees (Bombus spp.). The protocol only allows for a single exposure phase, so modifications based on Siviter, Matthews and Brown (In Preparation) were used to include an additional parasite exposure phase.

Worker bees were housed in Nicot cages a day in advance of parasite exposure, and then rank allocated to treatments based on weight, with an even distribution of source colonies by treatment. Bees outside the range of $0.1 \mathrm{~g}-0.4 \mathrm{~g}$ were not used. Syringes with $50 \%(\mathrm{w} / \mathrm{w})$ sucrose were added to the Nicot cages for sustenance. The tip of the syringe was clipped off to allow access to the sucrose.

The subsequent day, following the OECD 247 protocol (OECD, 2017), we exposed bees in the parasite treatments to an inoculum containing 10,000 cells of Crithidia bombi. The parasite inoculum was prepared by removing 40 worker bees from a $C$. bombi infected colony and inducing them to defecate. The faeces were then purified following Cole (1970). Purified $C$. bombi solution was then diluted in distilled water and mixed $1: 1$ with $50 \%(w / w)$ sucrose to produce the test solution with 10,000 cells in $40 \mu \mathrm{L}$ of inoculum. A control solution of 1:1 distilled water and $50 \%(\mathrm{w} / \mathrm{w})$ sucrose was also produced. Pilot work had demonstrated that 
182 this method leads to very high infection rates (>95\%). At dissection any bees with a parasite 183 intensity of 0 cells per $\mu \mathrm{L}$ were deemed to have a failed infection, and were excluded from the

184

185 186

187 188

202

203

204

205

206

207

208

209

210

211

212

213

214

215 experiment. A further single worker with an intensity of 100 cells per $\mu l$, which is more likely to have resulted from contamination of the slide than an infection, was also excluded.

Sucrose syringes were removed for 2-4 hours prior to exposure to the inoculum, starving the bees. Then $40 \mu \mathrm{L}$ of solution was pipetted into a fresh syringe and this was added to each cage. The bees were left to feed on the inoculum for a further four hours, at which point the syringe was removed and consumption visually verified. Bees that did not consume $>80 \%$ of the solution were excluded from the experiment. Bees were returned to ad libitum sucrose with a syringe of $50 \%(w / w)$ sucrose and had a small ball of pollen added ( 1g).

Bees were left for 7 days for the parasite infection to develop, at which point they entered the pesticide exposure phase. Here the above steps for parasite exposure were repeated, but with pesticide-laced treatment solutions replacing the parasite treatment solutions. The treatment doses used in all acute exposure experiment are listed in Table 1. below.

After exposure to the pesticide, mortality was recorded at four hours, 24 hours and 48 hours. Mortality was defined as a lack of response to physical agitation. Dead bees were discarded as their corpses degrade too quickly to be dissected.

Any bees who survived the full 48 hours were weighed (Scout SKX, Ohaus, Switzerland, accuracy limit of $0.001 \mathrm{~g}$ ), then transferred to a $2 \mathrm{~mL}$ Eppendorf tube and frozen at $-80 \mathrm{C}^{\circ}$ for later dissection. Bees in the $C$. bombi or C. bombi + Glyphosate treatment groups were later dissected. Bees were removed from the freezer and placed on ice. The abdomen was cut off and was pinned to a black wax plate. The abdomen was cut open on one side, and pinned open. $100 \mu \mathrm{L}$ of $0.8 \%$ Ringers solution was pipetted directly onto the gut to prevent desiccation and another $100 \mu \mathrm{L}$ onto the wax to the side of the body. The honey crop was cut, and the gut transferred to the droplet on the wax. The ileum was isolated and cut at both ends, with care to remove any Malpighian tubules and tracheal tissue. The ileum was moved to a $1.5 \mathrm{ml}$ Eppendorf with $100 \mu \mathrm{L}$ of $0.8 \%$ Ringers solution and ground using a pestle for five seconds in a set pattern of movements. The ground gut was then vortexed for a single second and $10 \mu \mathrm{L}$ pipetted onto a Neubauer haemocytometer slide and the $C$. bombi concentration counted. All endpoints are presented as mean \pm one standard deviation. 
216 Experiment one: Modified Ecotoxicological Protocol OECD 247: Small

\section{Scale}

218 In this initial exploratory experiment only the C. bombi only and Glyphosate $+C$. bombi

219 treatments were included. While bees were evenly allocated to treatments by colony of origin, 220 colony origin was not tracked through the experiment and as such this is not accounted for in 221 the statistics. Due to non-feeder events and deaths prior to the glyphosate exposure stage the 222 final treatment groups may have had an uneven allocation of colony of origin, although this is 223 unlikely due to the initial even distribution and low occurrence of such events. Sucrose 224 consumption was not measured.

225 Experiment two: Modified Ecotoxicological Protocol OECD 247: Full 226 Scale

227 This experiment was a full-scale repetition of experiment one, with all treatment groups 228 included. The Modified Ecotoxicological Protocol OECD 247 protocol described above was 229 followed with a single major deviation, in that haemolymph samples were taken from all bees 230 at the end of the experiment. The haemolymph was analysed as part of a different project. This 231 manipulation did not affect the mortality metric as mortality was recorded prior to the 232 manipulation. Further, it would not affect the parasite intensity measure as there is no by 233 treatment differences, and the timescale of the extraction is too short to influence $C$. bombi 234 levels. This experiment was conducted in two batches with just a single day stagger between 235 them.

Experiment three: Modified Ecotoxicological Protocol OECD 247: Long 237 Term Survival

238 To test for longer term effects a version of the Modified Ecotoxicological Protocol OECD 247 239 protocol described above was performed, with the only deviation being that bees were 240 maintained for 20 days post exposure rather than 48 hours. Mortality checks were made daily 241 and pollen balls renewed weekly.

\section{Experiment four: Microcolony Exposure- Acute Exposure}

243 To test for effects on reproduction a microcolony experiment was performed. Bees were 244 moved into microcolony boxes (clear acrylic boxes $(6.7 \times 12.7 \times 4.9 \mathrm{~cm})$, with a plastic mesh grate 245 bottom $(6.7 \times 7.3 \mathrm{~cm})$ ) a day prior to parasite exposure. Initially 8 workers per microcolony box 246 were added. 
248 Parasite inoculation and glyphosate exposure followed the Modified Ecotoxicological Protocol OECD 247, with bees being moved into Nicot cages for this exposure. Between treatments bumble bees were maintained in microcolony boxes.

251

252

Due to time constraints only bumble bees receiving a treatment were moved to Nicot cages and

253

254

255

256

257

258

259

260

261

262

263

264

265

266

267

268

269

270

271

272

273

274

275

276

277

278

279

280

281

282

283

284 exposed. Bees in the control treatment were never moved to Nicot cages, bees in the $C$. bombi only treatment and the glyphosate only treatment were moved to Nicot cages just once, and those in the Glyphosate $+C$. bombi treatment were moved to Nicot cages twice. This had the potential to cause a by treatment effect as being moved to a Nicot cage is a potentially stressful experience. However, the day prior to the $C$. bombi exposure day all bees were manipulated as they were moved from their source colony to a microcolony box. Similarly, on the glyphosate exposure day all bees not moved into Nicot cages were manipulated as they were moved into a fresh microcolony box. As such it is only the marginal additional level of stress from the time in the Nicot cages that could produce a by treatment effect. Bees in the Nicot cages were also kept in their microcolony box adjacent to nest-mates to reduce stress.

Non-feeders were excluded from the experiment at each of the exposure steps, which alongside mortality led to slightly lower worker numbers in the micro-colonies (Glyphosate: 6.9 \pm 1.2 , C. bombi: $6.7 \pm 1.1$, Glyphosate + C. bombi: $6.4 \pm 1.1$ (SD)), versus the control $(7.8 \pm 0.4$ $(\mathrm{SD})$ ). Workers who died $(n=4)$ or escaped $(n=5)$ during the experiment were recorded, but not replaced. This was accounted for in the analysis, however, with reproductive output expressed per worker present at end of experiment. Given that worker reproduction is highly dependent on the laying individual (Blacquière et al. 2012), this should robustly account for differing worker numbers.

After glyphosate exposure bumble bees were moved to a fresh microcolony box to reset their reproductive efforts, and then provided ad libitum sucrose and pollen for 14 days. 14 days is shorter than the time required for a bee to develop from egg to eclosion, so all adults at the end of the experiment were those initially added.

On day 14 , adult bumble bees were counted and frozen for later dissection to quantify parasite intensity, the total number of eggs and larvae number were counted, and total larval weight measured. Larval weight was chosen as the best measurement of reproductive success as it reflects output better than larval number. By using weight, the greater investment required to rear a L4 larvae, versus a L1 larvae, is reflected, whereas number of larvae would not reflect this investment disparity. As such larval weight per worker was chosen as the quantitative metric used for analysis. 


\section{Experiment five: Microcolony Exposure- Chronic Exposure}

286 This protocol is derived from the OECD 245 honey bee chronic oral toxicity test, with

287

288

289

290

291

292

293

294

295

296

297

298

299

300

301

302

303

304

305

306

307

308

309

310

311

312

313

314

315

316

317

318

319

320

321 modification to account for the different test species.

Workers used in the experiment were age controlled, to achieve this 8 workers were taken from a source colony, tagged and moved into a microcolony box. Pupae and enclosed larvae from the same colony were added, with the 8 tagged workers acting as nurses for them. Newly emerged workers were identified by their lack of a tag, and 10 days after the start of emergence they were moved to $\mathrm{Nicot}^{\circledR}$ cages for parasite inoculation. This inoculation followed the Modified Ecotoxicological Protocol OECD 247, with treatment groups detailed in Table 1. After excluding non-feeders, bees were then allocated to microcolonies in groups of six based on treatment, with all workers within a microcolony originating from the same source colony. Because the allocation to microcolonies occurred after non-feeders were excluded there is no by treatment exclusion effect. By selecting newly emerged workers over a 10-day period, workers were age controlled to be within 10 days of one another. Workers were left on ad libitum sucrose and pollen for a week while the parasite developed. After seven days the workers were moved to a fresh microcolony to reset their reproductive effort.

Data from Thompson et al. (2014) were used to inform the chronic exposure scenario. Thompson et al. (2014) measured glyphosate concentration in returning nectar and pollen from honey bees foraging on Phacelia tanacetifolia sprayed with a glyphosate-based herbicide formulation (MON 52276) according to full label restrictions. Using WebPlotDigitiser (Rohatgi, 2020), the values from Thompson et al. (2014)'s graphs were extracted. An inverse relationship model was used to model the declining residue concentration:

Glyphosate Concentration $=$ Intercept $+\frac{\text { Constant }}{\text { Time }}$. As the data from Thompson et al. (2014) has missing days and no data after 7 days, missing data were either interpolated or extrapolated. These modelled concentrations of returning nectar and pollen were then used to generate an exposure regime. Sucrose was fed to the bees ad libitum and was spiked with pesticides in concentrations shown in Figure 1. In all treatments $50 \% \mathrm{w} / \mathrm{w}$ sucrose was changed daily, and the previous day's consumption was recorded. The glyphosate concentration provided decreased over time with the modelled values. Degradation of the glyphosate will have occurred in the sucrose; however, this is largely insignificant given glyphosate's long halflife of 47-267 days (as measured in seawater) (Mercurio et al. 2014). $5 \mathrm{~g}$ of pollen was provided and in glyphosate treatments this was spiked with an average concentration of glyphosate over the 10 days exposure $(110 \mathrm{mg} / \mathrm{kg})$. This was done as changing pollen daily was not feasible, and $5 \mathrm{~g}$ was used as this amount was rarely wholly consumed by a group of workers in 14 days. In the positive control, the dimethoate concentration was maintained at a constant $1 \mathrm{mg} / \mathrm{L}$, and 
322 pollen was not spiked in this treatment. Following OECD 245 for honey bees (OECD, 2017a),

323 exposure ended on day 10, and all bumble bees were fed unspiked sucrose for another four 324 days. On day 14 bumble bees were frozen and reproductive output measured, as described

325 above. Mortality was recorded daily.

326

327

As the dataset used to calculate our chronic exposure regime was from a semi-field exposure

328 studied conducted in honey bees (Thompson et al. 2014), the use of these data for $B$. terrestris may be problematic. There are no comparable data from honey bees and bumble bees to be able to see if the same spraying regime leads to similar returning nectar concentrations.

331 However, as the only available dataset it is the best choice to inform the chronic exposure regime.

\section{Statistical testing}

334

335

336

337

338

339

340

341

342

343

344

345

346

347

348

349

350

351

352

353

354

355

356

Statistical analyses were carried out in ' $R$ ' programming software version 3.6.2 ( $R$ Core Team 2019) following the same analysis scheme as Straw, Carpentier and Brown, (2021). All plots were made using 'ggplot2' version 3.2.1 (Wickham, 2016) and 'survminer' version 0.4.6 (Kassambara, Kosinski \& Biecek, 2019). AIC model simplification was used, with conditional model averaging where no single model had $>95 \%$ AIC support. The candidate set of models was chosen by adding the next best supported model until a cumulative $>95 \%$ AIC support was reached. 'MuMIn' version 1.43.17 was used for model averaging (Bartoń 2020). Parameter estimates and 95\% confidence intervals are reported. 'Ime4' version 1.1-23 was used for Linear Mixed Effects models (Bates et al. 2020) and 'coxme' version 2.2-16 was used for Mixed Effects Cox Proportional Hazards models (Therneau, 2020). Confidence intervals not crossing zero indicate a significant effect, so a confidence interval of -1.00 to 1.00 would not be significant, but a confidence interval of -2.00 to -1.00 would be. Model assumptions were checked graphically and using statistical testing, including using 'e1071' version 1.7-4 (Mayer et al. 2021). Model parameters, AIC weights and final models are presented in Supplementary Tables 6-11.

\section{Experiment one: Modified Ecotoxicological Protocol OECD 247 Small Scale}

Parasite intensity: Data were found to be non-normal using a Shapiro-Wilks test, so a Kruskal Wallis test was used with the model (Parasite Intensity Treatment).

Mortality: Due to an absence of mortality in the experiment no statistical testing was conducted.

\section{Experiment two: Modified Ecotoxicological Protocol OECD 247 Full Scale}


357 Parasite intensity: Data were found to be non-normal using a Shapiro-Wilks test, so a Kruskal

358

359

360

361

362

363

364

365

366

367

368

369

370

371

372

373

374

375

376

377

378

379

380

381

382

383

384

385

386

387

Wallis test was used with the model (Parasite Intensity Treatment).

Mortality: Due to an absence of mortality in the experiment, except in the positive control where all bees died, no statistical testing was conducted.

\section{Experiment three: Modified Ecotoxicological Protocol OECD 247: Long Term Survival}

Mortality: A Cox Proportional Hazards model was used to analyse the mortality data. Due to the near complete mortality in the positive control treatment, this treatment was excluded from the mortality analysis as it violates the proportionality of hazards assumption. The full model used was (Mortality Treatment + Body Weight $+(1 \mid$ Colony)). Proportionality of hazards was checked graphically.

\section{Experiments four and five: Microcolony Exposure- Acute Exposure and Chronic Exposure}

Reproduction: Larval weight, adjusted to the number of workers present at the end of the experiment, was found to be non-normal using a Shapiro-Wilks test. It was accordingly square root transformed, and confirmed to be normal using a further Shapiro-Wilks test. The full model used was (Larval Weight per Worker Treatment + Body Weight of Initial Workers + Number of Workers Alive at the End of the Experiment + (1|Colony)).

Parasite intensity: A Linear Mixed Effect model was used to analyse the parasite intensity data. The full model used was (Parasite Intensity Treatment + (1|Micro Colony ID) + (1|Colony)).

\section{Acute Exposure Only:}

Mortality: Mortality was too low to allow a Linear model, Linear Mixed Effects models, or ChiSquare test. Accordingly, a Fishers Exact test was used with the model (Survival Treatment).

\section{Chronic Exposure Only:}

Sucrose/Glyphosate consumption: A Linear Mixed Effect model was used to analyse the Sucrose Consumption data. The full model used was (Sucrose Consumption Treatment * Time + Weight of Bees at Start of Exposure + (1|Micro Colony ID) + (1|Colony)).

Mortality: Mortality was too low to allow a Linear model, Linear Mixed Effects models, or ChiSquare test. Accordingly, a Fishers Exact test was used with the model (Survival Treatment). 
388

389

390

391

392

393

394

395

396

397

398

399

400

401

402

403

404

405

406

407

408

409

410

411

412

413

\title{
Results
}

\section{Modified Ecotoxicological Protocol OECD 247: Small Scale}

\author{
Parasite intensity
}

The Glyphosate $+C$. bombi treatment had a significantly higher parasite intensity than the $C$. bombi only treatment (Kruskal-Wallis $X^{2}(1)=7.885, p=0.005$ ). Glyphosate $+C$. bombi treated bees $(n=21)$ had an average parasite intensity of $14,519 \pm 10,462$ (SD) cells per $\mu \mathrm{L}$ compared to $6,946 \pm 5,682$ cells per $\mu \mathrm{L}$ in the $C$. bombi only treatment $(n=23)$ (Figure 2 ).

\section{Mortality}

No mortality was observed in either the $C$. bombi, or the Glyphosate $+C$. bombi treatment.

\section{Modified Ecotoxicological Protocol OECD 247: Full Scale}

\begin{abstract}
Parasite intensity
In contrast to the first experiment, Glyphosate $+C$. bombi did not have a significantly different parasite intensity to the $C$. bombi only treatment (Kruskal-Wallis $X^{2}(1)=0.42818, p=0.5129$ ). Glyphosate $+C$. bombi treated bees $(n=34)$ had an average parasite intensity of $24,124 \pm$ 14,664 cells per $\mu \mathrm{L}$, compared to the $20,756 \pm 14,473$ cells per $\mu \mathrm{L}$ in the $C$. bombi only treatment $(n=32)$ (see Figure 3). Neither body weight or batch had a significant effect on parasite intensity (Linear Mixed Effect model: parameter estimate (PE) =66,940.7, 95\% Cl [-19,878.3 to $152,664.5]$ and $(\mathrm{PE})=897.3,95 \% \mathrm{Cl}[-6,843.0$ to 8,512.8] respectively).
\end{abstract}

\section{Mortality}

No mortality was observed in any treatment bar the positive control, where all bees died within 24 hours.

\section{Modified Ecotoxicological Protocol OECD 247: Long Term Survival}

\section{Mortality}

All bees in the positive control treatment, bar one, died within two days, while all other treatments experienced mortality over the 20-day period. 
414 C. bombi only, Glyphosate only, and Glyphosate + C. bombi did not have significantly different 415 mortality compared to the negative control (Cox proportional hazards mixed effects model: 416 parameter estimate $(P E)=0.728,95 \% \mathrm{Cl}[-0.81$ to 0.96$],(P E)=1.27,95 \% \mathrm{Cl}[-0.92$ to 1.18$]$, and $417 \mathrm{PE}=1.19,95 \% \mathrm{Cl}$ [-0.89 to 1.14], respectively). C. bombi only, Glyphosate only, and Glyphosate $418+C$. bombi had $4 \%, 7 \%$ and $6 \%$ mortality respectively, while the control had $2 \%$ mortality (see 419 Figure 4), a real terms difference of one to two bees.

420

There was no significant difference in reproductive output between treatments. While the mean larval weight per worker ( \pm SD and number of microcolonies) varied between treatments $(0.510 \mathrm{~g} \pm 0.224, n=8$ in the control, $0.458 \mathrm{~g} \pm 0.349, n=11$ in the $C$. bombi only treatment, $0.405 \pm 0.141, n=9$ in the Glyphosate only treatment and 0.339g $\pm 0.224, n=10$ in the Glyphosate $+C$. bombi treatment (see Figure 5)), a null model, which contained the response variable, the co-variate of initial worker weight and the random colony variable, but not the treatment variable, was the best supported model with $\geq 95 \%$ AIC support. This model found a significant effect of Original Weight of Nurse Workers on reproductive output (Linear mixed effects model (LMER) $=0.26,95 \% \mathrm{Cl}[0.14$ to 0.37$])$, with heavier workers being more successful at rearing offspring.

Glyphosate $+C$. bombi exposed bees did not have a significantly different parasite intensity to the $C$. bombi only treatment (Linear Mixed Effect model: parameter estimate (PE) = -314.6, 95\% $\mathrm{Cl}[-2,865.81$ to $2,236.55])$. Glyphosate $+C$. bombi treated bees $(n=64)$ had an average parasite intensity of $18,362 \pm 7,704$ cells per $\mu \mathrm{L}$, compared to the $18,635 \pm 5,884$ cells per $\mu \mathrm{L}$ in the $C$. bombi only treatment $(n=74)$ (see Figure 6).

439 Mortality

440 There was no significant difference in mortality by treatment (Fisher Exact test (two sided) $p=$ 441 0.679). C. bombi only, Glyphosate only and Glyphosate + C. bombi had 1\%, $0 \%$ and $3 \%$ mortality 442 respectively, while the control had $2 \%$ mortality, a real terms difference of one bee. 


\section{Experiment five: Microcolony Exposure- Chronic Exposure}

\section{Reproduction}

445 There was no significant difference in reproductive output between treatments. The mean

446 larval weight per worker ( \pm SD and number of microcolonies) varied between treatments, with $4470.106 \mathrm{~g} \pm 0.077, n=8$ in the control, $0.053 \mathrm{~g} \pm 0.054, n=8$ in the $C$. bombi only treatment, $0.143 \mathrm{~g}$ $448 \pm 0.139, n=8$ in the Glyphosate only treatment and 0.124g $\pm 0.103, n=8$ in the Glyphosate $+C$. 449 bombi treatment (see Figure 7). The model average with a cumulative $\geq 95 \%$ AIC support did not 450 include the treatment term. The two models included were both null models, one with the co451 variate of initial worker weight and random colony variable, and the second with just the 452 random colony variable. This model found no significant effect of Original Weight of Nurse 453 Workers on reproductive output (Linear mixed effects model (LMER) $=0.20,95 \% \mathrm{Cl}[-0.15$ to 454 0.27]).

455 456

457

458 459

460

461

462

463

464

465

466

467

468

469

470

471

472

473

474

475

476

\section{Sucrose/Glyphosate consumption}

Over the 10-day exposure period the average consumption of sucrose per worker was $5.890 \pm$ $0.676 \mathrm{~mL}$ in the control, $5.880 \pm 0.865 \mathrm{~mL}$ in the $C$. bombi only treatment, $5.947 \pm 0.875 \mathrm{~mL}$ in the Glyphosate only treatment, and $6.271 \pm 0.746 \mathrm{~mL}$ in the Glyphosate $+C$. bombi treatment.

The model average that contained models with a cumulative $\geq 95 \%$ AIC support did not include the Treatment term. As such Treatment had no effect on sucrose consumption. The weight of the bees at the start of exposure also did not affect sucrose consumption, (Linear Mixed Effect model: parameter estimate $(\mathrm{PE})=0.062,95 \% \mathrm{Cl}[-0.052$ to 0.069$])$.

Over the 10-day exposure period the average consumption of glyphosate per worker was $38.7 \pm$ $5.4 \mu \mathrm{g}$ in the Glyphosate only treatment, and $41.4 \pm 4.3 \mu \mathrm{g}$ in the Glyphosate $+C$. bombi treatment. The majority of this consumption was in the initial few days, as the concentration decreased markedly over time. Figure 8 shows the sharp decline in glyphosate consumption over time.

Parasite intensity

Glyphosate $+C$. bombi did not have a significantly different parasite intensity to the $C$. bombi only treatment (Linear Mixed Effect model: parameter estimate (PE) $=1649.0,95 \% \mathrm{Cl}[-3251.24$ to 6529.72$]$ ). Glyphosate $+C$. bombi treated bees $(n=42)$ had an average parasite intensity of $20,562 \pm 7065$ cells per $\mu \mathrm{L}$ compared to $18,759 \pm 9403$ cells per $\mu \mathrm{L}$ for the $C$. bombi only treatment $(n=44)$ (see Figure 9). 
477 Mortality

478 All bees in the positive control died. There was no significant difference in mortality between 479 the remaining treatments (Fisher Exact test (two sided) $p=0.903$ ). C. bombi only, Glyphosate 480 only and Glyphosate $+C$. bombi had $0 \%, 2 \%$ and $2 \%$ mortality respectively, while the control 481 had $4 \%$ mortality, a real terms difference of one to two bees. 


\section{Discussion}

483 Through a series of experiments, we show no robust evidence for the effects of either

484 glyphosate, $C$. bombi, or their combination, on mortality or a range of sublethal effects (sucrose 485 consumption, parasite intensity and reproduction) in bumble bees. Acute exposure to either 486 stressor or their combination over a range of timescales representing the majority of a bee's 487 lifespan did not cause mortality, nor did chronic exposure over a 10-day period. While an initial 488 experiment found an acute dose of $200 \mu \mathrm{g}$ of glyphosate caused a considerable increase in the 489 intensity of the parasite $C$. bombi, this effect was not seen in any of the follow up experiments. 490 We found no evidence to suggest glyphosate affects reproduction among workers, and, 491 contrary to predictions from previous studies (Shykoff \& Schmid-Hempel 1991, Brown, Loosli 492 and Schmid-Hempel 2000), no evidence that $C$. bombi does either.

493

494

495

496

497

498

499

500

501

502

503

504

505

506

507

508

509

510

511

512

513

514

515

516

517

\section{Mortality}

The most basic metric of bee health is mortality. A dead bee can contribute nothing further to its fitness, as it is unable to contribute to the provisioning of brood or production of sexuals. Most regulatory systems use mortality as the initial metric to assess toxicity (EFSA 2012, EFSA 2013, EPA, 2014). In the EU, lower tier testing considers just acute contact and oral toxicity in honey bees (EFSA 2012, EFSA 2013), and bumble bees (including OECD 247 studies), although the addition of bumble bee data has not yet been fully implemented (EFSA 2015). In the case of glyphosate, the $\mathrm{LD}_{50} \mathrm{~S}$ derived were found to be above the threshold value of $200 \mu \mathrm{g}$ active ingredient per bee (or equivalent highest possible tested dose)(EFSA, 2015), although this was only done with honey bees, as bumble bee data are not due to be submitted until the $2025 \mathrm{EU}$ renewal of glyphosate. As such, glyphosate was not entered into higher tier testing, meaning that from a regulatory testing standpoint only short-term mortality was considered (EFSA, 2015). This was used to justify the current lack of any mitigation measures for exposure of bees to glyphosate or glyphosate-based herbicides.

The data presented here supports the regulatory conclusion that glyphosate does not cause mortality in the short term (EFSA, 2015). These data also expand the species upon which we have evidence of the mortality effects of glyphosate, with the addition of a bumble bee to the previously studied honey bee. Our results show no mortality over a range of exposures and time periods from 2-20 days, going well beyond the two-day test regulators will conduct on bumble bees using OECD 247. Additionally, there were no mortality effects from the interaction between glyphosate, with either acute or chronic exposure, and C. bombi in worker bumble bees. It is important to clarify that our experiments used glyphosate as an active ingredient, not as a formulation. 
518 Several experiments have tested glyphosate-based herbicide formulations, as opposed to the 519 active ingredient glyphosate, on honey bees (Abraham et al. 2018, Faita et al. 2020, Odemer et 520 al. 2020 and Motta et al. 2020) and non-Apis bees (Ruiz-Toledo and Sánchez-Guillén, 2014, 521 Abraham et al. 2018, Seide et al. 2018, Straw, Carpentier and Brown, 2021). However, co522 formulants in glyphosate-based herbicides can have significant effects on toxicity (Motta et al. 523 2020, Straw, Carpentier and Brown, 2021), making these studies difficult to interpret from the 524 perspective of the active ingredient. Consequently, the following discussion of existing 525 academic literature will be limited to experiments that solely test the active ingredient 526 glyphosate.

527

528 In line with our results, the academic literature has largely found no evidence for effects of 529 glyphosate on adult honey bee worker survival. Over a range of concentrations up to $210 \mathrm{mg} / \mathrm{kg}$, 530 and across a range of timelines, no significant mortality has been observed in multiple studies 531 (Herbert et al. 2014, Goñalons and Farina 2018, Motta, Raymann and Moran 2019, Blot et al. 532 2019). Yet, despite these results, Almasri et al. (2020) found that just $0.00083 \mathrm{mg} / \mathrm{kg}$, a 533 concentration approximately 2 million times lower than $210 \mathrm{mg} / \mathrm{kg}$, significantly reduced 534 survival over 20 days. It is not clear from Almasri et al.'s (2020) methods if the solvent dimethyl 535 sulfoxide was present in the control treatment, which could potentially have confounded the 536 results. Interestingly, Almasri et al. (2021) failed to replicate this result using the same 537 concentration. Further, Motta and Moran, (2020) found that concentrations as low as $5389.625 \mathrm{mg} / \mathrm{kg}$ caused significant mortality over 20-40 days. However, neither Almasri et al. (2020) 539 or Motta and Moran, (2020) report screening their honey bees for parasites prior to the trial.

540 While a recent meta-analysis of the mortality effects of glyphosate on bees suggested a 541 significant effect of glyphosate on mortality (Battisti et al. 2021), the methods used heavily 542 predisposed the results to confirm the mortality hypothesis (Straw 2021), In addition, errors in 543 the data extraction process and analysis mean that the conclusions drawn in this meta-analysis 544 lack support (Straw 2021).

545

546 Interestingly, work on honey bees has not been limited to adult workers, honey bee larvae have 547 also been tested. In honey bees, evidence for mortality in larvae is heavily mixed. Tomé et al.

548 (2020) found that six days of exposure to $0.054 \mathrm{mg} / \mathrm{kg}$, but not $0.0008 \mathrm{mg} / \mathrm{kg}$, caused significant 549 mortality at 18 days after treatment started, although the authors note that the $16 \%$ mortality 550 is 'considered incidental because [their methodology] accepts up to $30 \%$ control mortality'. 551 Vazquez et al. (2018) had very mixed results over five days exposure, with their highest 552 treatment group $5 \mathrm{mg} / \mathrm{kg}$ causing significant mortality in one colony, but no change in four 553 colonies, and significantly reduced mortality in one colony. Dai et al. (2018) found that over 21 554 days exposure to $4 \mathrm{mg} / \mathrm{kg}$ or $20 \mathrm{mg} / \mathrm{kg}$ caused significant mortality, but that $0.8 \mathrm{mg} / \mathrm{kg}$ did not. 
556 These mixed results, for both adults and larvae, heavily indicate strong colony effects, or that

557

558

559

560

561

562

563

564

565

566

567

568

569

570

571

572

573

574

575

576

577

578

579

580

581

582

583

584

585

586

587

588

589

590

591

592

some bees were infected with a parasite, like Serratia marcescens, which synergises with glyphosate to cause mortality (Motta, Raymann and Moran, 2018, Motta et al. 2020). Notably, none of these studies, in adults or larvae, explicitly reported screening their bees for signs of disease. Thompson et al. (2014) used verifiably healthy bees making it the most robust study to date. They found that over 15 days of exposure neither $75 \mathrm{mg} / \mathrm{L}, 150 \mathrm{mg} / \mathrm{L}$ or $301 \mathrm{mg} / \mathrm{L}$ caused any larval mortality. This highlights the importance of screening bees for diseases prior to experiments, as well as the need for more work to understand the effects of pesticides on parasite exposed bees. Odemer et al. (2020) also found no evidence of mortality in a range of experiments (adults and larvae) using parasite-free honey bees, but, as noted above, these results are not directly comparable because of the use of a glyphosate-based formulation (although glyphosate co-formulants are linked to increased, not reduced, toxicity (Mesnage, Bernay and Séralini 2012, Nagy et al. 2021)).

The larval mortality literature relates to the experiments presented here because the larvae in the chronic exposure experiment will also have been fed glyphosate by the nurse workers. However, in our experimental paradigm the peak exposure for micro-colonies would have occurred while new offspring were still in the egg stage. Our results did not explicitly consider larval mortality, but no effect was seen on larval number or weight (consistent with Thompson et al. (2014)), which indicates that if any mortality occurred it was below the level required to reduce reproductive success. Further experiments, where peak exposure occurs at the larval feeding stage, are required to understand whether results from honey bee larvae extrapolate to bumble bee larvae. Larvae were not the primary subjects of this study, adult workers were, and as such the evidence collected on their mortality is more substantial.

In the short term (two days) and long term (20 days) after exposure to a relatively high acute dose of glyphosate, no mortality was seen in individually housed bees in three separate experiments (Modified Ecotoxicological Protocol OECD 247: Small Scale, Full Scale and LongTerm Mortality). As 20 days is representative of a considerable proportion of a bumble bee worker's lifespan (Brian, 1952, Rodd, Plowright and Owen, 1980, Goldblatt and Fell, 1987), this indicates that there is no delayed mortality response and no meaningful shortening of longevity. All the academic studies cited above have used chronic exposure to glyphosate, not acute exposure. As such, there is presently no non-regulatory data on acute exposure to glyphosate in any bee species, nor any data on glyphosate exposure in bumble bees, so our results represent a substantive contribution to the understanding of glyphosate's effects on bee mortality.

Peer) reviewing PDF | (2021:08:64998:1:1:NEW 19 Oct 2021) 
593 In the microcolony experiments no significant mortality was seen with either adult workers

594 acutely exposed, or age controlled young adult workers with chronic exposure. This

595 demonstrates that even while the bees are housed collectively under more natural conditions,

596 and exerting themselves rearing young, any potential stress was insufficient to cause mortality.

597 The finding of no mortality with a fully field realistic chronic exposure regime in parasite free

598 bumble bees supports the evidence that chronic glyphosate exposure is non-lethal to healthy

599 worker bees (Herbert et al. 2014, Goñalons and Farina 2018, Motta, Raymann and Moran 2019,

600 Blot et al. 2019). The lack of increased mortality alongside $C$. bombi infection also aids our

601 understanding of which parasites can synergise with glyphosate to cause mortality in bees.

602 Mortality, however, is not the only metric of bee health, and other sublethal metrics like

603 parasite intensity are important to consider for a more complete picture of bee health.

\section{Parasite intensity.}

605 The initial experiment found a 109\% increase in C. bombi intensity. As a preliminary experiment

606 the methods were less robust than later experiments, with a smaller sample size and no

607 tracking of colony of origin or body weight through the experiment. However, the balanced

608 experimental design accounts for this variation and as such it is unlikely to be confounded.

609 Further the sample size of $C$. bombi $n=21$ and Glyphosate $+C$. bombi $n=23$ is appropriately

610 powered (Logan, Ruis-González and Brown, 2005).

611

612 The follow up experiment to this, found a $16 \%$ increase in C. bombi intensity, although this

613 effect was not statistically significant. In this trial the sample size was larger, and the co-variates

614 of colony of origin and body weight were tracked throughout.

615

616 These opposing results can be explained in several ways. Principally either of the two

617 experiment could have delivered a false positive or a false negative result, which is the simplest

618 solution, and there is no evidence to confirm or contradict this. Alternatively, it is possible that

619 some of the other variables in the experiment such as the parasite, the colonies used, or other

620 unknown effects are acting individually or in combination to alter the parasite intensity.

621

622 As with all bumble bee toxicity testing the colonies used differed between experiments.

623 Because of this, there could be a parasite by host genotype interaction (Baer and Schmid-

624 Hempel, 2003), or a parasite by host microbiome interaction (Koch and Schmid-Hempel, 2011,

625 Mockler et al. 2018) as has been observed in experiments previously. However, we believe that

626 this is unlikely as in each experiment three or more colonies were used to account for inter-

627 colony variation, these were evenly distributed to treatment groups, and colonies were sourced

628 from the same supplier. 
629

630 More interestingly, the two experiments differed heavily in the average parasite intensity in the 631 C. bombi only treatments. While the first experiment had a parasite intensity of $6,946 \pm 5,682$

632 cells per $\mu \mathrm{L}(\mathrm{SD})$, the follow up experiment had a mean parasite intensity of $20,756 \pm 14,473$

633 cells per $\mu \mathrm{L}$, which is considerably higher. It is possible that this increase in parasite intensity 634 reached a plateau, meaning any increase in parasite intensity caused by glyphosate could no 635 longer occur, as there was no further scope for intensity to rise. To assess the evidence for this 636 hypothesis we can look to prior C. bombi literature.

637

638 The majority of the literature on C. bombi in B. terrestris uses faecal counts, which are not 639 directly comparable to homogenised gut counts. Further, there are currently no comparable 640 data on peak parasite intensity using homogenised gut counts. As such it is not possible to 641 know if the levels seen in our second experiment do represent a plateau. However, our 642 methods were based on unpublished work (Siviter, Matthews and Brown, In Preparation), that 643 found a mean parasite intensity of 1,849 1 1,966 cells per $\mu \mathrm{L}$, comparable to levels in our first 644 experiment, but more than ten times lower than intensity levels in our second experiment. This 645 is indicative that a plateau may have been reached. Similarly, in our microcolony experiments,

646 which took place in between the two experiments on individual bumblebees, a high parasite 647 intensity (Acute: $18,635 \pm 5,884$ cells per $\mu \mathrm{L}$ and Chronic: $18,759 \pm 9403$ cells per $\mu \mathrm{L}$ ) was 648 recorded.

649

Faecal parasite counts from Logan, Ruiz-González and Brown, (2005) found parasite intensity to 651 rise to a peak at around 13 days post inoculation. So, at 9 days post-exposure the parasite intensity should not have plateaued. However, in all experiments after our first small scale experiment, the parasite intensities at either 9 days or 21 days were in the 20,000 cells per $\mu \mathrm{L}$

654 range. This again supports the plateau hypothesis because there is a consistent and high parasite intensity across a range of experiments and conditions.

656

If the $C$. bombi intensity had reached a plateau, that such high parasite intensities do not cause

658

659

660

661

662

663

664

665

666 any measurable impacts on the other metrics recorded under the conditions tested here does indicate that even if glyphosate does increase parasite intensity, this is not likely to lead to any reduction in fitness. As such any effect that might exist is unlikely to be environmentally relevant or robust.

A final explanation for these conflicting results may come from the parasite source used. The $C$. bombi used in the experiments was from the same original source, wild caught infected $B$. terrestris spring queens. Faeces collected from infected queens were used to infect a commercial colony which were kept as a parasite source. As each commercial colony neared 
667 the end of its lifespan, faeces was collected from workers in it and used to infect a new

668 commercial colony. Theoretically, within a year the serial passage of the parasite could lead to 669 selection for higher infection levels, and if this were the case it could explain our experimental

670 results. However, previous work with C. bombi suggests that the opposite occurs, with serial

671 passage within a colony reducing infectivity to non-colony members (Yourth and Schmid-

672 Hempel, 2006), which would result in lower prevalence and intensity of infections in our

673 experimental paradigm, a pattern we did not see. Consequently, it seems unlikely that an

674 increase in transmissibility or growth in C. bombi across the course of experiments can explain

675 our results. While parasite intensity is an important factor in bee health, reproductive success is

676 much more important to a bee's fitness.

\section{Reproduction}

678 Reproductive success is the ultimate metric of bee health, directly representing bee fitness.

679 Drone production by unmated workers in a microcolony set up is designed to function as a 680 proxy of this, and itself does not directly represent a field realistic measure of whole colony 681 sexual production. There is even some evidence that microcolonies can give contradictory 682 results to queenright laboratory or full field experiments (Oystaeyen et al. 2020). As such our 683 results should be interpreted with caution, and are not a field realistic measure of reproductive 684 success.

685

686

687

688

689

690

691

692

693

694

695

696

697

698

699

700

701

No significant effect on reproduction was found in any experiment, despite at times large differences between treatments (up to a $33.5 \%$ difference in reproductive success versus the control), which is potentially indicative of power limitation. Indeed, it is possible that both microcolony experiments were power limited, with 10 microcolonies per treatment (a total of 38 and 36 microcolonies in each experiment). This is less than other microcolony experiments like Oystaeyen et al. (2020) which used 20 per treatment, and Siviter et al. (2019) which used 30 per treatment. The power limitation hypothesis is supported by the lack of a significant effect of $C$. bombi on reproductive success in both experiments, which contrasts with a range of published literature (Yourth, Brown and Schmid-Hempel 2008, Shykoff and Schmid-Hempel, 1991, Brown, Schmid-Hempel, and Schmid-Hempel 2003). Interestingly, while not significant, $C$. bombi reduced reproductive success by $10.2 \%$ and $50.0 \%$ in the Acute and Chronic experiments respectively. This is a similar scale of reduction to previously published data (Brown, SchmidHempel, and Schmid-Hempel 2003). The data presented here also indicate that acute exposure to glyphosate is more likely to impact reproductive success than chronic exposure, with a $20.6 \%$ decline in reproductive success after acute exposure, versus a $34.9 \%$ increase after chronic exposure. Overall, we would suggest that this evidence be used to guide future studies, 
702 conducted ideally in field conditions with larger sample sizes to provide more high quality and

703 definitive evidence for any potential effects.

704

705

706

There was a considerably lower reproductive output overall in the Chronic experiment than in

707 the Acute exposure experiment. This is likely because the workers in the Chronic exposure

708 experiment were age controlled, and thus likely to be much younger on average. This could have led to a delay in ovary development retarding reproductive output. In the Chronic

710 exposure experiment, sucrose consumption was also tracked to allow for the total glyphosate exposure to be measured.

\section{Sucrose}

712 Sucrose consumption can be an indicator of bee health (Straw and Brown, In Review). While in

713

714

715

716

717

718

719

720

721

722

723

724

725

726

727

728

729

730

731

732

733

734

735

736

737 isolation this metric has no clear relation to fitness, the ultimate measure of bee health, it can be useful in indicating that a bee is acting abnormally. In the case of exposure to the coformulant alcohol ethoxylates, reduced sucrose consumption went hand in hand with weight loss and gut melanisation (Straw and Brown, In Review). Further, sucrose consumption could be a corollary of pollination services, as bees with lower appetites might forage less, although in social bees nectar foraging is a response to both individual and colony-level nectar needs (Hendriksma, Toth and Shafir, 2019). Under chronic exposure, no treatment affected sucrose consumption, indicating that glyphosate did not significantly affect the bees dietary consumption.

Under microcolony conditions worker bees consumed an average of 38.7 or $41.4 \mu \mathrm{g}$ of glyphosate (Glyphosate and Glyphosate + C. bombi treatments respectively) under a field realistic, degrading concentration exposure regime. This can be used to inform future research as to the cumulative exposure bees would experience in the wild. The majority of this glyphosate was consumed within the first few days of exposure, with the rapidly declining residues causing the consumption from day five onwards to contribute little to overall exposure. Consequently, future studies could truncate the glyphosate exposure to five days with little reduction in exposure. However, it is also worth noting that there is no limit on the number of sprays of a glyphosate-based herbicide per year, or a mandated time gap between them (Roundup ProActive Label), so repeat exposure could occur. As such, the 38.7 or $41.4 \mu \mathrm{g}$ dose does not necessarily represent the total dose a bee could be exposed to over their lifetime.

The stepwise degradation method of exposure, as developed for bees in Linguadoca et al. (2021), is the most field realistic existing method of simulating real pesticide exposure in a 
738 laboratory setting. By mimicking the degradation of the substance the exposure profile is

739 accurately portrayed, whereas a flat exposure, even using a time-weighted average dose, would

740 lack the nuance of the initial peak followed by a lengthy tail. As such, the lack of mortality

741 resulting from this chronic exposure can be seen as a very rigorous result, representing the best

742 approximation of the effects of a field realistic exposure possible.

743

744 The research presented here principally used acute oral exposure to $200 \mu \mathrm{g}$ of glyphosate as an

745 active ingredient. None of the research into the effects of glyphosate on the honey bee

746 microbiome has used acute exposure, instead using chronic exposure at a range of

747 concentrations from $0.8 \mathrm{mg} / \mathrm{kg}$ (Dai et al. 2018) to $210 \mathrm{mg} / \mathrm{kg}$ (Blot et al. 2019). It is possible that

748 sustained exposure to glyphosate is more impactful than a single more concentrated instance

749 of exposure because the gut microbial community is not afforded opportunity to recover.

750 Alternatively, exposure to the considerably higher acute concentration may also have a more

751 severe impact, potentially acting to cull sensitive species and strains. Given that bees are

752 exposed to both acute and chronic exposure to glyphosate in the wild, if future research

753 considered acute exposure our understanding of how glyphosate affects bee health would be

754 more complete.

755

756

How the acute exposure to $200 \mu \mathrm{g}$ of glyphosate used in this study relates to in-field exposure is

757 unknown. There is no data, even from honey bees, to be able to accurately predict acute

758 exposure to herbicides that lack any mitigation measures. Given that flowering weeds can be

759 sprayed while bees are foraging on them, and glyphosate is typically sprayed in very

760 concentrated sprays (compared with insecticides), for a bee to consume $200 \mu \mathrm{g}$ in a short period

761 of time immediately after a spray application is not implausible, although lower doses are more likely. More work on acute exposure of bees to agrochemicals without bee specific mitigation measures is needed to inform future research. However, with no effects on a range of metrics seen at this potentially high-end dose, it is likely that more field realistic acute exposures would also not have an effect on bumble bees. 


\section{Conclusions}

767 As the world's most used pesticide (Duke and Powles, 2008, Benbrook, 2016), the application of 768 glyphosate is a hotly debated topic, largely due to its human carcinogenicity (Alcántara-de la 769 Cruz et al. 2021), but increasingly regarding its potential toxicity to bees (Cullen et al. 2019).

770 Given its wide usage, the implications for changing its regulatory status would substantively

771 reshape conventional farming practices (Beckie, Flower and Ashworth, 2020), and thus need to

772 be made using robust and environmentally sound science. As such it is imperative that evidence

773 for or against its impacts on bees is of the highest of standards.

774

775

With that in mind, the findings presented here provide robust evidence that oral exposure to

776 the active ingredient glyphosate does not induce mortality in the bumble bee $B$. terrestris. We

777 report mixed evidence for the effect of glyphosate on $C$. bombi parasite intensity, with

778 insufficient evidence to describe the effect as environmentally robust. While future research

779 could elucidate the impacts of glyphosate on C. bombi intensity, as we found no effects in any metric of their combination, research efforts are best focussed on other pesticide-parasite combinations. Further we report no effects of glyphosate, C. bombi or their combination on worker reproductive output, but this conclusion is potentially limited by the power of the study. Our results thus do not indicate any requirement to change the regulatory status of the active ingredient glyphosate as it pertains to bumble bees. As glyphosate has been found to impact honey bees as measured by a range of sublethal metrics (Boily et al. 2013, Herbert et al. 2014, Balbuena et al. 2015, Helmer et al. 2015, Vazquez et al. 2018), further research using wild bee species and sublethal metrics would help resolve whether this widely used chemical is safe for 788 bees. 


\section{Acknowledgements}

790 Thanks to A. Linguadoca for his help with the project and J. R. G. Adams for his help interpreting 791 the microbiome literature. 
792 References

793 Abraham, J., Benhotons, G.S., Krampah, I., Tagba, J., Amissah, C., and Abraham, J.D. (2018).

794

795

796

797

798

799

800

801

802

803

804

805

806

807

808

809

810

811

812

813

814

815

816

817

818

819

820

821

822

823

824

825

826

827

828 Commercially formulated glyphosate can kill non-target pollinator bees under laboratory conditions. Entomologia Experimentalis et Applicata, 166, 695-702.

Adler, L.S., Michaud, K.M., Ellner, S.P., McArt, S.H., Stevenson, P.C. and Irwin, R.E. 2018. Disease where you dine: plant species and floral traits associated with pathogen transmission in bumblebees. Ecology, 99, 2535-2545.

Alcántara-de la Cruz, R., Cruz-Hipolito, H.E. Alfredo Domínguez-Valenzuela, J., and De Prado, R. (2021). Glyphosate ban in Mexico: potential impacts on agriculture and weed management. Pest Management Science, 77, 3820-3831.

Almasri, H., Tavares, D.A., Diogon, M., Pioz, M., Alamil, M., Sené, D., Tchamitchian, S., Cousin, M., Brunet, J-C., and Belzunces, L.P. (2021). Physiological effects of the interaction between Nosema ceranae and sequential and overlapping exposure to glyphosate and difenoconazole in the honey bee Apis mellifera. Ecotoxicology and Environmental Safety, $217,112258$.

Almasri, H., Tavares, D.A., Pioz, M., Sené, D., Tchamitchian, S., Cousin, M., Brunet, J.L., and Belzunces, L.P. (2020). Mixtures of an insecticide, a fungicide and a herbicide induce high toxicities and systemic physiological disturbances in winter Apis mellifera honey bees. Ecotoxicology and Environmental Safety. 203, 111013.

Baer, B., and Schmid-Hempel, P. (2003). Bumblebee workers from different sire groups vary in susceptibility to parasite infection. Ecology Letters, 6, 106-10.

Balbuena, M.S., Tison, L., Hahn, M.L., Greggers, U., Menzel, R., and Farina, W.M. (2015). Effects of sublethal doses of glyphosate on honeybee navigation. Journal of Experimental Biology, 218, 2799-2805.

Baron, G.L., Raine, N.E., and Brown, M.J.F. (2014). Impact of chronic exposure to a pyrethroid pesticide on bumblebees and interactions with a trypanosome parasite. Journal of Applied Ecology, 51, 460-69.

Bartoń, K. MuMIn: multi-model inference. R package version 1.0.0. https://CRAN.Rproject.org/package=MuMIn (2020).

Bates, D., Mächler, M., Bolker, B., and Walker, S. (2015) Fitting linear mixed-effects models using Ime4. Journal of Statistical Software, 67, 1-48.

Battisti, L., Potrich, M., Sampaio, A.R., de Castilhos Ghisi, N., Costa-Maia, M., Abati, R., dos Reis Martinez, C.B., and Sofia, S.H., (2021). Is glyphosate toxic to bees? A meta- analytical review. Science of the Total Environment, 767, 145397.

Beckie. H.J., Flower, K.C., and Ashworth, M.B. (2020). Farming without glyphosate? Plants, 9, 1-15. Benbrook, C.M. (2016). Trends in glyphosate herbicide use in the United States and globally. Environmental Science Europe, 28, 1-15. 
829 Blacquière, T., Smagghe, G., van Gestel, C.A.M., and Mommaerts, V. (2012). Neonicotinoids in bees: a

830

831

832

833

834

835

836

837

838

839

840

841

842

843

844

845

846

847

848

849

850

851

852

853

854

855

856

857

858

859

860

861

862

863

864

865 review on concentrations, side-effects and risk assessment. Ecotoxicology, 21, 973-992.

Blot, N., Veillat, L., Rouzé, R., and Delatte, H. (2019). Glyphosate, but not its metabolite AMPA, alters the honeybee gut microbiota. PLoS One, 14, e0215466.

Boily, M., Sarrasin, B., DeBlois, C., Aras, P., and Chagnon, M. (2013). Acetylcholinesterase in honey bees (Apis mellifera) exposed to neonicotinoids, atrazine and glyphosate: laboratory and field experiments. Environmental Science and Pollution Research, 20, 56035614.

Brian, A. D. (1952). Division of labour and foraging in Bombus agrorum Fabricius. Journal of Animal Ecology, 21, 223-240.

Brown, M.J.F., Loosli, R. and Schmid-Hempel, P. (2000). Condition-dependant expression of virulence in a trypanosome infecting bumblebees. Oikos, 91, 421-27.

Brown, M.J.F., Schmid-Hempel, R., and Schmid-Hempel, P. (2003). Strong context-dependent virulence in a host-parasite system: reconciling genetic evidence with theory. Journal of Animal Ecology, 72, 994-1002.

Cisarovsky, G., and Schmid-Hempel, P. (2014). Combining laboratory and field approaches to investigate the importance of flower nectar in the horizontal transmission of a bumblebee parasite. Entomologia Experimentalis et Applicata, 152, 209-15.

Cullen, M.G., Thompson, L.J., Carolan, L.C., Stout, J.C. and Stanley, D.A. (2019). Fungicides, herbicides and bees: A systematic review of existing research and methods. PLoS ONE, 14, e0225743.

Dai, P., Yan, Z., Ma, S., Yang, Y., Wang, Q., Hou, C., Wu, Y., Liu, Y., and Diao, Q. (2018). The herbicide glyphosate negatively affects midgut bacterial communities and survival of honey bee during larvae reared in vitro. Journal of Agricultural and Food Chemistry, 66, 7786-93.

Duke, S.O., and Powles, S.B. 2008. Glyphosate: A Once-in-a-Century Herbicide. Pest Management Science, 64, 319-25.

Duke, S.O. (2018). The history and current status of glyphosate. Pest Management Science, 74, 10271034.

Durrer, S., and Schmid-Hempel, P. (1994). Shared use of flowers leads to horizontal pathogen transmission. Proceedings of the Royal Society of London B: Biological Sciences, 258, 299302.

EFSA. (2012). Scientific Opinion on the science behind the development of a risk assessment of Plant Protection Products on bees (Apis mellifera, Bombus spp. and solitary bees). EFSA Journal, 10, 2668.

EFSA. (2013). EFSA Guidance document on the risk assessment of plant protection products on bees (Apis mellifera, Bombus spp. and solitary bees). EFSA Journal, 11, 3295.

EFSA. (2015). Conclusion on the peer review of the pesticide risk assessment of the active substance glyphosate. EFSA Journal, 13, 4302. 
866

867

868

869

870

871

872

873

874

875

876

877

878

879

880

881

882

883

884

885

886

887

888

889

890

891

892

893

894

895

896

897

898

899

900

901

902

903

EPA. (2014). Guidance for assessing pesticide risks to bees. Office of Pesticide Programs, Washington DC.

Faita, M.R., Cardozo, M.M., Amandio, D.T.T., Orth, A.I., and Nodari, R.O., 2020. Glyphosatebased herbicides and Nosema sp. microsporidia reduce honey bee (Apis mellifera L.) survivability under laboratory conditions. Journal of Apicultural Research, 59, 332-342.

Farina, W.M., Balbuena, M.S., Herbert, L.T., Megoni Goñalons, C., and Vázquez, D.E. (2019). Effects of the herbicide glyphosate on honey bee sensory and cognitive abilities: individual impairments with implications for the hive. Insects, 354, 1-16.

Fauser-Misslin, A., Sadd, B.M., Neumann, P., and Sandrock, C. (2014). Influence of combined pesticide and parasite exposure on bumblebee colony traits in the laboratory. Journal of Applied Ecology, 51, 450-59.

Figueroa, L.L., Blinder, M., Grincavitch, C., Jelinek, A., Mann, E.K., Merva, L.A. Metz, L.E., Zhao, A.Y., Irwin, R.E., McArt, S.H., and Adler, L.S. (2019). Bee pathogen transmission dynamics: deposition, persistence and acquisition on flowers. Proceedings of the Royal Society B., 286, 20190603.

Folly, A.J., Koch, H., Stevenson, P.C., and Brown, M.J.F. (2017). Larvae act as a transient transmission hub for the prevalent bumblebee parasite Crithidia bombi. Journal of Invertebrate Pathology, 148, 81-85.

Gillespie, S. 2010. Factors affecting parasite prevalence among wild bumblebees. Ecological Entomology, 35, 737-47.

Goldblatt J.W., and Fell, R.D. (1987). Adult longevity of workers of the bumble bees Bombus fervidus (F.) and Bombus pennsylvanicus (De Geer) (Hymenoptera: Apidae). Canadian Journal of Zoology, 65, 2349-2353.

Gonalons, C.M., and Farina, W.M. (2018). Impaired associative learning after chronic exposure to pesticides in young adult honey bees. Journal of Experimental Biology, 221, 176644.

Graystock, P., Yates, K., Evison, S.E.F., Darvill, B., Goulson, D., and Hughes, W.O (2013). The Trojan hives: pollinator pathogens, imported and distributed in bumblebee colonies. Journal of Applied Ecology, 50, 1207-1215.

Helmer, S.H., Kerbaol, A., Aras, P., Jumarie, C., and Boily, M., (2015). Effects of realistic doses of atrazine, metolachlor, and glyphosate on lipid peroxidation and diet-derived antiox-idants in caged honey bees (Apis mellifera). Environmental Science and Pollution Research, 22, 8010-8021.

Hendriksma, H.P., Toth, A.L., and Shafir, S. (2019). Individual and colony level foraging decisions of bumble bees and honey bees in relation to balancing of nutrient needs. Frontiers in Ecology and Evolution, 7, 177.

Herbert, L.T., Vázquez, D.E., Arenas, A., and Farina, W.M. (2014). Effects offield-realistic doses of glyphosate on honeybee appetitive behaviour. Journal of Experimental Biology, 217, 3457-3464. 
904 Hicks, B. J., Pilgrim, B.L., Perry, E., and Marshall, H.D. (2018). Observations of native bumble bees inside 905 of commercial colonies of Bombus impatiens (Hymenoptera: Apidae) and the potential for 906 pathogen spillover. Canadian Entomologist, 150, 520-31.

907 Jones, C.M. and Brown, M.J.F. (2014). Parasites and genetic diversity in an invasive bumble bee. Journal 908 of Animal Ecology, 83, 1428-1440.

909

910

911

912

913

914

915

916

917

918

919

920

921

922

923

924

925

926

927

928

929

930

931

932

933

934

935

936

937

938

939

940

Kassambara, A., Kosinski, M. and Biecek, P. survminer: drawing survival curves using 'ggplot2'. R package version 0.4.6. https://CRAN.R-project.org/package=survminer (2019).

Koch, H., and Schmid-Hempel, P. (2011). Socially transmitted gut microbiota protect bumble bees against an intestinal parasite. Proceedings of the National Academy of Sciences, 108, 19288-92.

Koch, H., Woodward, J., Langat, M.K., Brown, M.J.F., and Stevenson, P.C. (2019) Flagellum removal by a nectar metabolite inhibits infectivity of a bumblebee parasite. Current Biology, 29, 3494-3500.

Korner, P., and Schmid-Hempel, P. (2005). Correlates of parasite load in bumblebees in an Alpine habitat. Entomological Science, 8, 151-160.

Linguadoca, A., Rizzi C., Villa, S., and Brown, M.J.F. (2021). Sulfoxaflor and nutritional deficiency synergistically reduce survival and fecundity in bumblebees. Science of the total environment, 795, 148680.

Logan, A., Ruiz-González and Brown, M.J.F. (2005). The impact of host starvation on parasite development and population dynamics in an intestinal trypanosome parasite of bumble bees. Parasitology, 130, 637-642.

Mayer, D. Dimitriadou, E., Hornik, K., Weingessel, A., Leisch, F., Chang, C.-C., and Lin, C.-C. (2021). Misc Functions of the Department of Statistics, Probability Theory Group (Formerly: E1071), TU Wien. R package version 1.7-4. https://cran.rproject.org/web/packages/e1071/index.html

Mercurio, P., Flores, F., Mueller, J.F., Carter, S., and Negri, A.P. (2014). Glyphosate persistence in seawater. Marine Pollution Bulletin, 85, 385-390.

Mesnage, R., Bernay, B., and Séralini, G-E. (2013). Ethoxylated adjuvants of glyphosate-based herbicides are active principles of human cell toxicity. Toxicology, 313, 122-128.

Mockler, B.K., Kwong, W.K., Moran, N.A., and Koch, H. (2018). Microbiome structure influences infection by the parasite Crithidia bombi in bumble bees. Applied and Environmental Microbiology, 83, e02335-17.

Motta, E.V.S., and Moran, N.A. (2020). Impact of glyphosate on the honey bee gut microbiota: effects of intensity, duration, and timing of exposure. mSystems, 5, e00268-20.

Motta, E.V.S., Mak, M., De Jong, T.K., Powell, J.E., O’Donnell, A., Suhr, K.J., Riddington, I.M., and Moran, N.A. (2020). Oral or topical exposure to glyphosate in herbicide formulation impacts the gut microbiota and survival rates of honey bees. Applied Environmental Microbiology, 86, se0115020. 
941 Motta, E.V.S., Raymann, K., and Moran, N.A. (2018). Glyphosate perturbs the gut microbiota of honey 942 bees. Proceedings of the National Academy of Sciences, 115, 10305-10310.

943 Nagy, K., Duca, R. C., Lovas, S., Creta, M., Scheepers, P. T. J., Godderis, L., and Ádám, B. (2020).

944 Systematic review of comparative studies assessing the toxicity of pesticide active ingredients and

945

946

947

948

949

950

951

952

953

954

955

956

957

958

959

960

961

962

963

964

965

966

967

968

969

970

971

972

973

974

975

976

977

978 their product formulations. Environmental Research, 181, 108926.

Odemer, R., Alkassab, A.T., Bischoff, G., Frommberger, M., Wernecke, A., Wirtz, I.P., Pistorius, J., and Odemer, F. (2020). Chronic high glyphosate exposure delays individual worker bee (Apis mellifera I.) development under field conditions. Insects, 11, 1-20.

OECD. (2017a). Honey Bee (Apis mellifera L.), Chronic oral toxicity test (10-day feeding). OECD Guidelines for the Testing of Chemicals, 245, 1-9

OECD. (2017b). Bumblebee, acute oral toxicity test. OECD Guidelines for the Testing of Chemicals, 247, 1-12

Oystaeyen, A.V., Klatt, B.K., Petit, C., Lenaerts, N., and Wäckers, F. (2020). Short-term lab assessments and microcolonies are insufficient for the risk assessment of insecticides for bees. Chemosphere, 273, 128518.

R Development Core Team. (2019). R: a language and environment for statistical computing. $R$ Foundation for Statistical Computing. https://www.R-project.org.

Rodd, F.H., Plowright, R.C., \& Owen, R.E. (1980). Mortality rates of adult bumble bee workers (Hymenoptera: Apidae). Canadian Journal of Zoology, 58, 1718-1721.

Rohatgi, A. (2020). WebPlotDigitizer. Version 4.4. https://automeris.io/WebPlotDigitizer

Roundup ${ }^{\circledR}$ ProActive Label. (2019). Retrieved from https://www.greentech.co.uk/media/shared/chemicallabels/Roundup\%20Pro\%20Active\%20360\%20Product\%20Label-min.pdf

Ruiz-Toledo, J., and Sánchez-Guillén, D. (2014). Effect of the concentration of glyphosate present in bodywaters near transgenic soybean fields on the honeybee Apis mellifera,and the stingless bee Tetragonisca angustula. Acta zoológica Mexico, 30, 408-413.

Rutrecht, S.T., and Brown, M.J.F. (2008). The life-history impact and implications of multiple parasites for bumble bee queens. International Journal for Parasitology, 38, 799-808.

Schmid-Hempel, P., Puhr, K., Kruger, N., Reber, C., and Schmid-Hempel, R. (1999). Dynamic and genetic consequences of variation in horizontal transmission for a microparasitic infection. Evolution, 53, 426- 34.

Seide, V.E., Bernardes, R.C., Pereira, E.J.G., and Lima, M.A.P. (2018). Glyphosate is lethal and cry toxins alter the development of the stingless bee Melipona quadrifasciata. Environmental Pollution, 243, 1854-1860.

Shykoff, J. A., and Schmid-Hempel, P. 1991. Incidence and effects of four parasites in natural populations of bumble bees in Switzerland. Apidologie, 22, 117-25.

Siviter, H., Horner, J., Brown, M.J.F., and Leadbeater, E. (2020) Sulfoxaflor exposure reduces egg laying in bumblebees Bombus terrestris. Journal of Applied Ecology, 57, 160-169. 
979 Straub, L., Strobl, V., and Neumann, P. (2020). The need for an evolutionary approach to ecotoxicology.

$980 \quad$ Nature Ecology \& Evolution, 4, 895.

981

Straw, E.A., Carpentier, E.N., Brown, M.J.F. (2021). Roundup causes high levels of mortality following

983 contact exposure in bumble bees. Journal of Applied Ecology, 58, 1167-1176.

984

Straw, E.A. (2021). Letter to the editors regarding Battisti et al (2021): Is glyphosate toxic to bees? A

985 meta-analytical review. Science of the Total Environment, 790, 147556.

986

Therneau, T.M. A package for survival analysis in R. Version 3.1-11. https://CRAN.R-

987 project.org/package=survival (2020).

Thompson, H.M., Levine, S.L., Doering, J., Norman, S., Manson, P., Sutton, P., and von Mérey, G. (2014).

988

989 Evaluating exposure and potential effects on honeybee brood (Apis mellifera) development using

990 glyphosate as an example. Integrated Environmental Assessment and Management, 10, 463-470.

Tomé, H.V.V., Schmehl, D.R., Wedde, A.E., Godoy, R.S.M., Ravaiano, S.V., Guedes, R.N.C.,

991 Martins, G.F., and Ellis, J.D. (2020). Frequently encountered pesticides can cause multiple

992 disorders in developing worker honey bees. Environmental Pollution, 256, 113420.

993

Vázquez, D.E., Ilina, N., Pagano, E.A., Zavala, J.A., and Farina, W.M. (2018). Glyphosate affects the larval 994

995 development of honey bees depending on the susceptibility of colonies. PLoS One, 13, 1-19.

996

Wickham, W. ggplot2: elegant graphics for data analysis. Springer-Verlag New York. https://CRAN.Rproject.org/package=ggplot2 (2016).

997

Yourth, C. P., and Schmid-Hempel, P. (2006). Serial passage of the parasite Crithidia bombi within a 998 colony of its host, Bombus terrestris, reduces success in unrelated hosts. Proceedings of the Royal Society B: Biological Sciences 273 (1587): 655-59.

1001

Yourth, C.P., Brown, M.J.F., and Schmid-Hempel, P. (2008). Effects of natal and novel Crithidia bombi (Trypanosomatidae) infections on Bombus terrestris hosts. Insectes Sociaux, 55, 86- 90. 


\section{Table $\mathbf{1}$ (on next page)}

Table 1. Showing the doses of parasite or pesticide given to each worker in a given treatment. 


\begin{tabular}{|l|l|l|}
\hline Control & $\begin{array}{l}\text { C. bombi only } \\
10,000 \text { cells per worker }\end{array}$ & $\begin{array}{l}\text { Positive control } \\
4 \mu g \text { dimethoate per } \\
\text { worker }\end{array}$ \\
\hline Glyphosate only & $\begin{array}{l}\text { Glyphosate and C. bombi } \\
10,000 \text { cells per worker } \\
200 \mu \text { g per worker }\end{array}$ & \\
\hline
\end{tabular}

1 Table 1. Showing the doses of parasite or pesticide given to each worker in a given treatment. 2 


\section{Figure 1}

Chronic exposure profile

Figure 1. Showing a stepwise chronic exposure profile for nectar generated from Thompson et al. (2014). With glyphosate concentration (in $\mathrm{mg} / \mathrm{kg}$ ) presented on the $\mathrm{Y}$ axis and time in days on the $\mathrm{X}$ axis. 


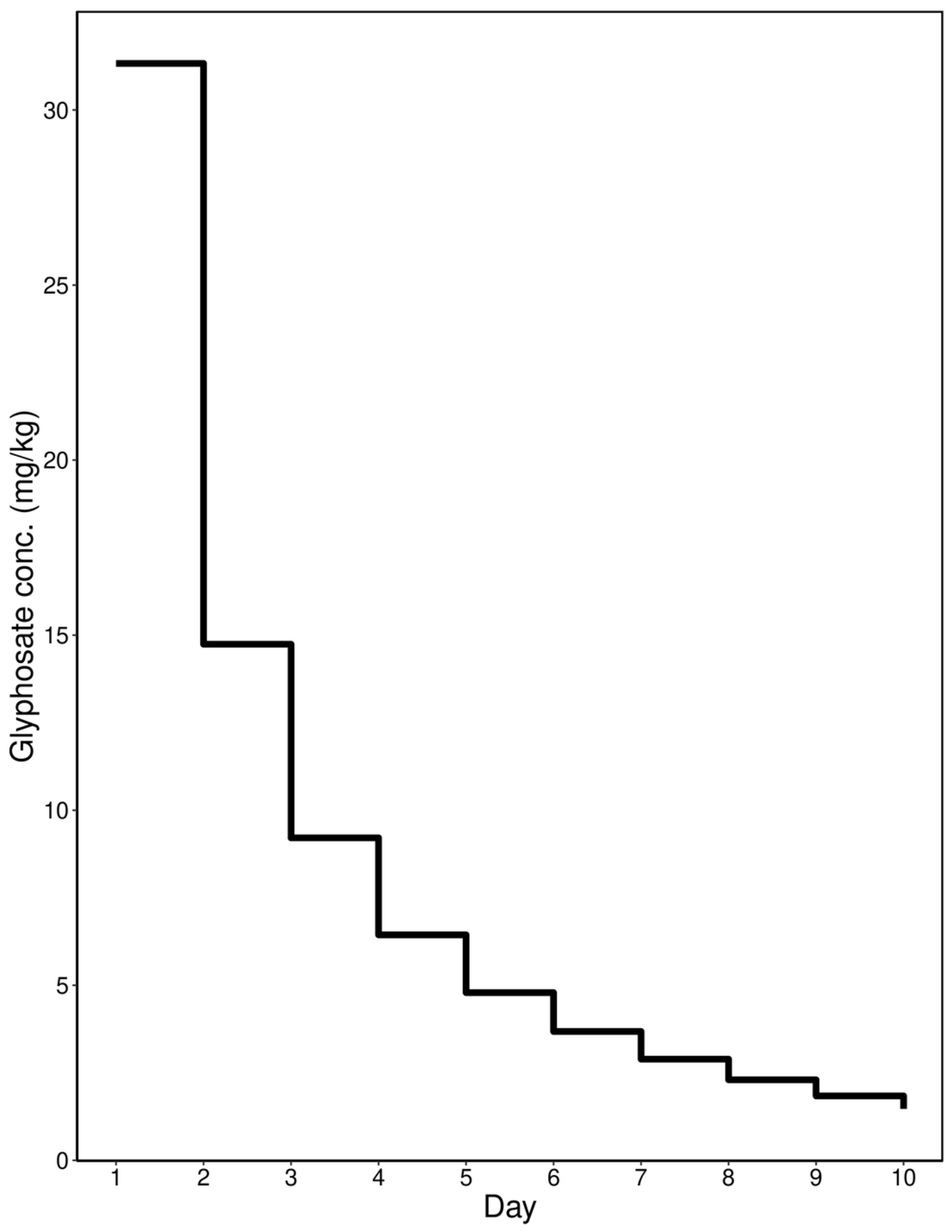


Figure 2

Modified Ecotoxicological Protocol OECD 247: Small Scale- Parasite Intensity

Figure 2. A boxplot with overlaid jittered data points showing the parasite intensity by treatment. 


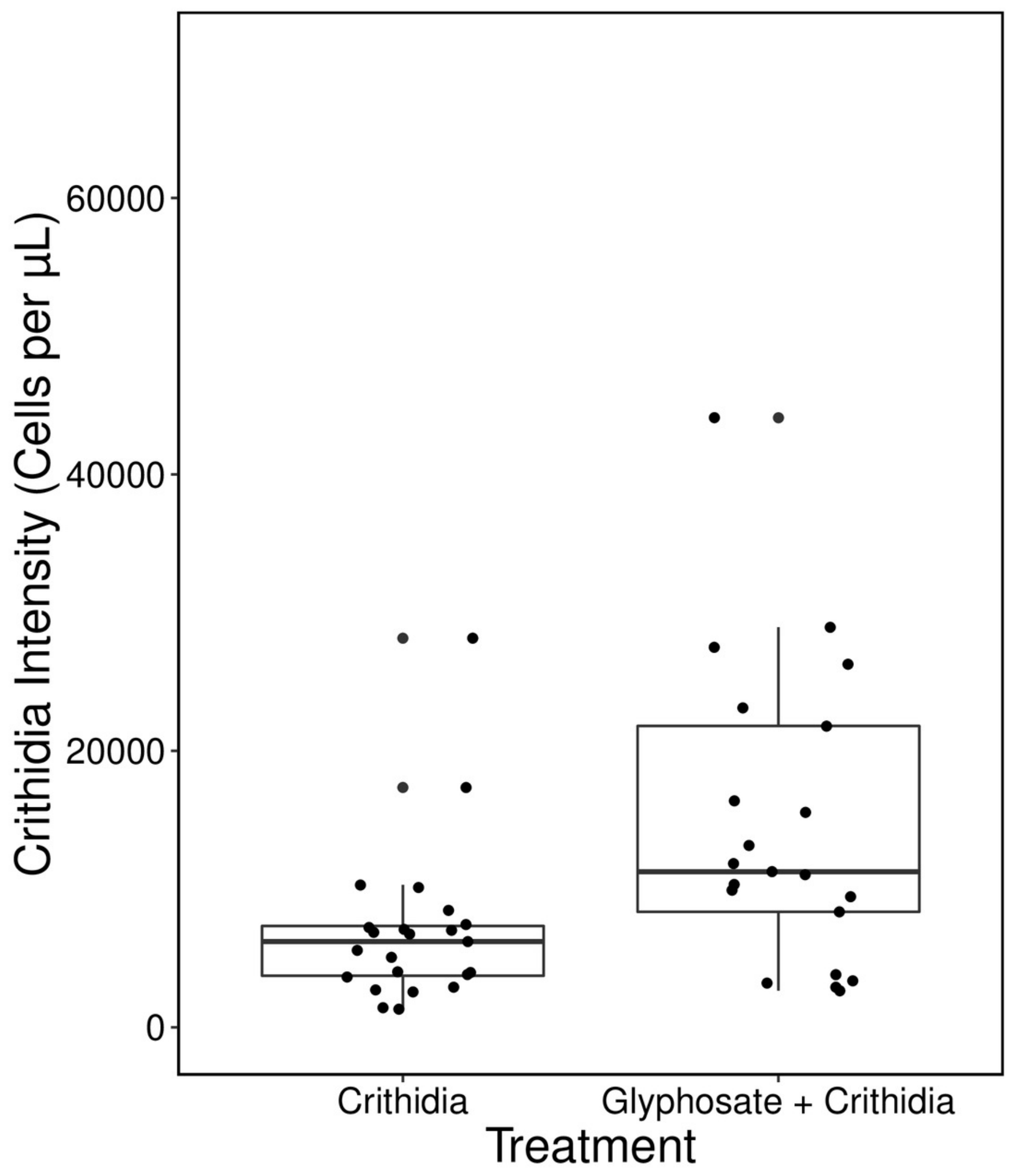


Figure 3

Modified Ecotoxicological Protocol OECD 247: Full Scale - Parasite Intensity

Figure 3. A boxplot with overlaid jittered data points showing the parasite intensity by treatment. 


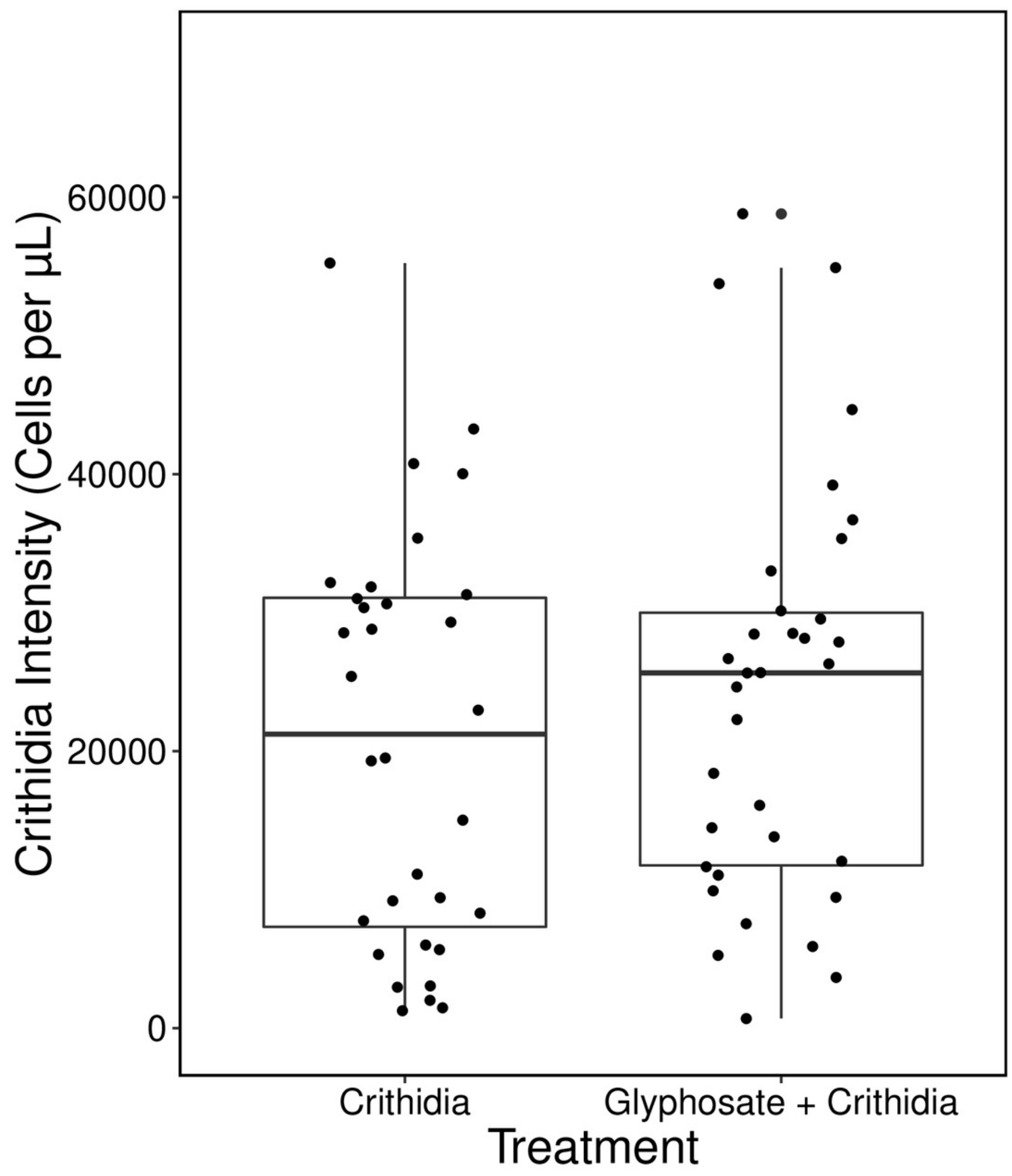


Figure 4

Long term survival after acute exposure

Figure 4. A Kaplan-Meier plot showing the survival over time by treatment. 


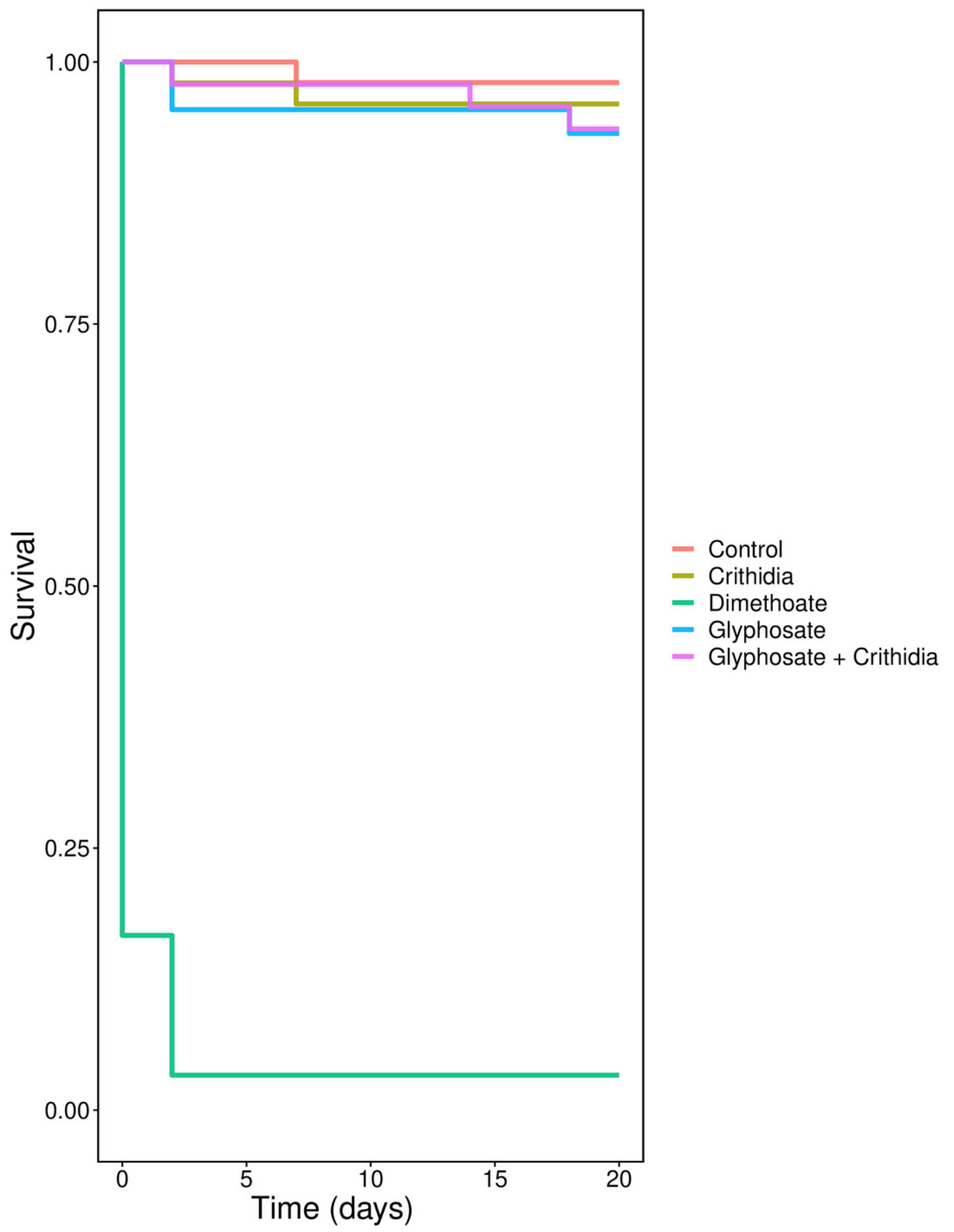




\section{Figure 5}

Larval weight adjusted for worker number after acute exposure

Figure 5. A boxplot showing the larval weight per microcolony standardised by the number of workers, presented by treatment with overlaid jittered data points. 


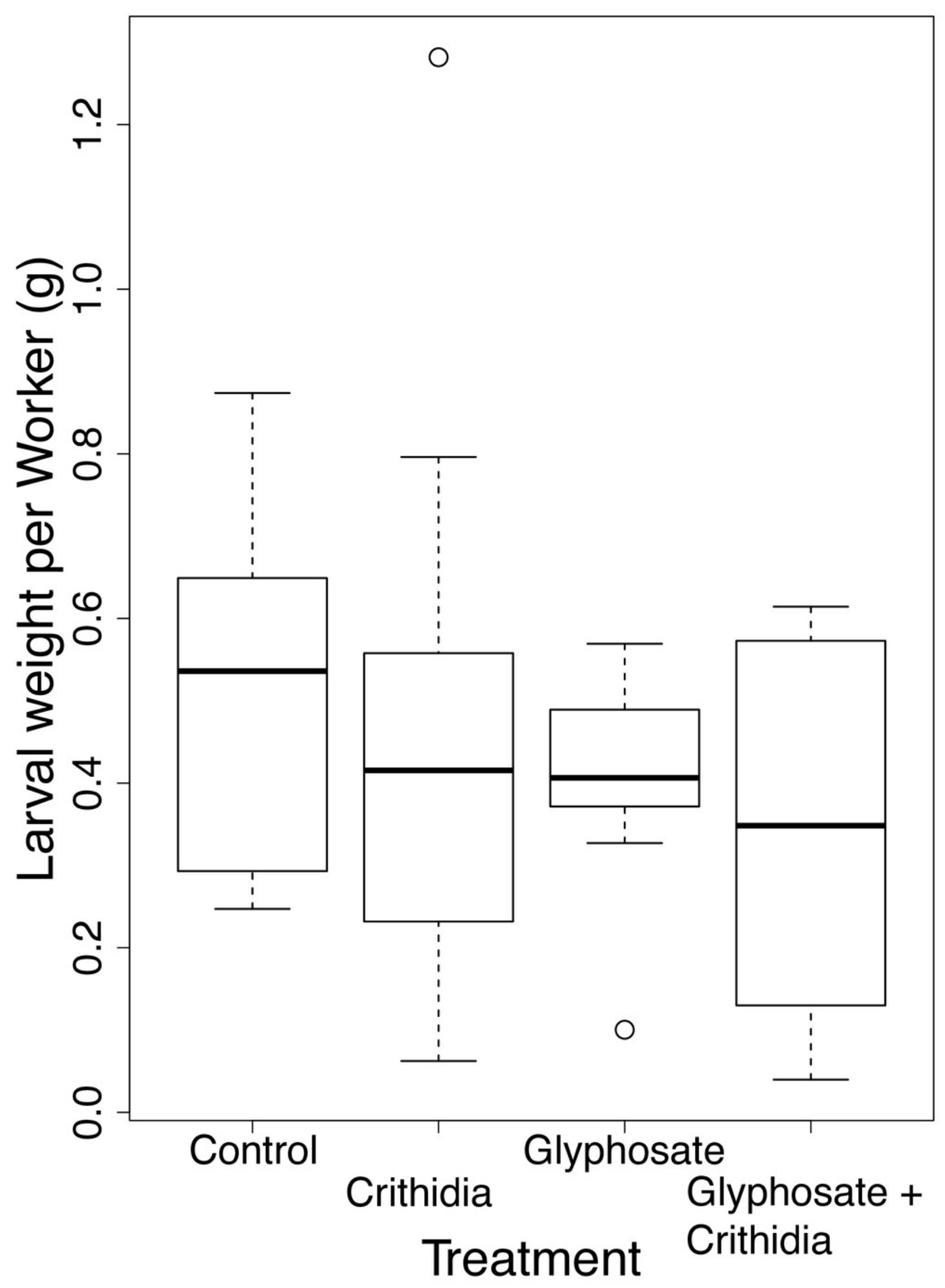


Figure 6

Microcolony Exposure - Acute Exposure - Parasite Intensity

Figure 6. A boxplot with overlaid jittered data points showing the parasite intensity by treatment. 


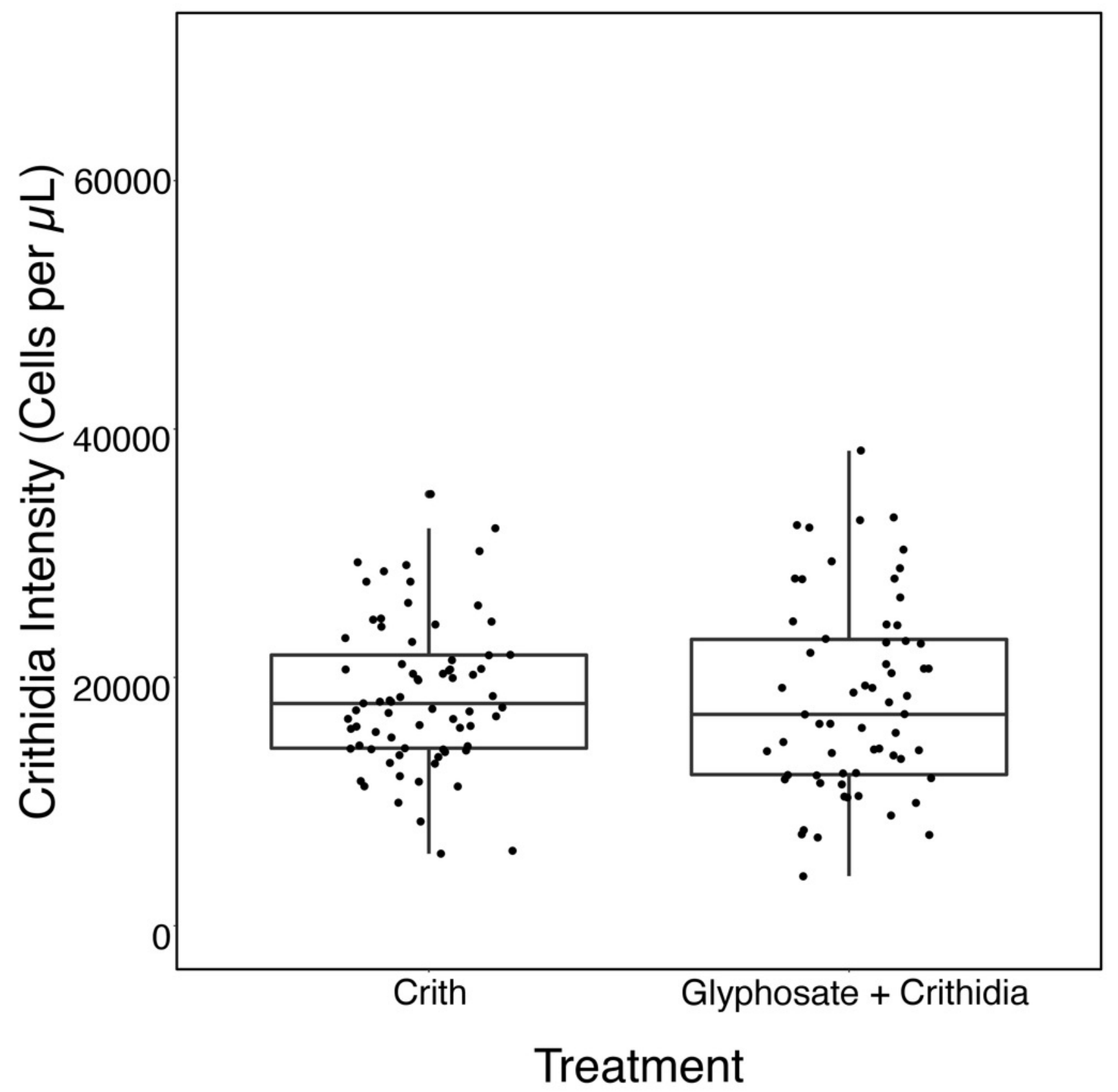




\section{Figure 7}

Larval weight adjusted by worker number after chronic exposure

Figure 7. A boxplot showing the larval weight per microcolony standardised by the number of workers, presented by treatment with overlaid jittered data points. All bees in the positive control died, accordingly they produced no larvae. 


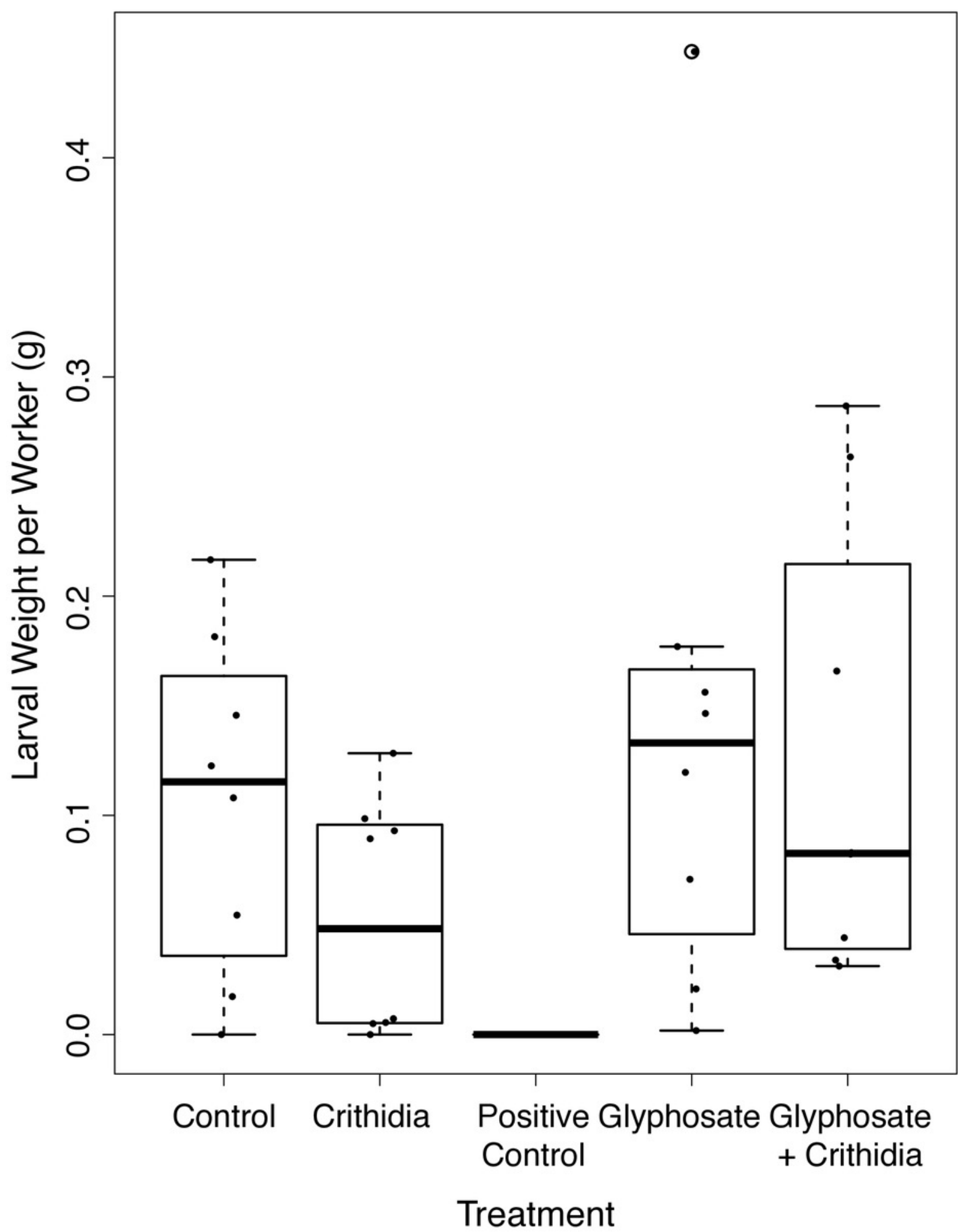




\section{Figure 8}

Glyphosate consumption over chronic exposure period

Figure 8. A scatter plot showing the daily consumption of the active ingredient glyphosate over time, presented by treatment. Data points have been horizontally jittered for clarity. Bees in the Control and C. bombi only treatments had glyphosate exposures of zero, and have been omitted from the graph. 


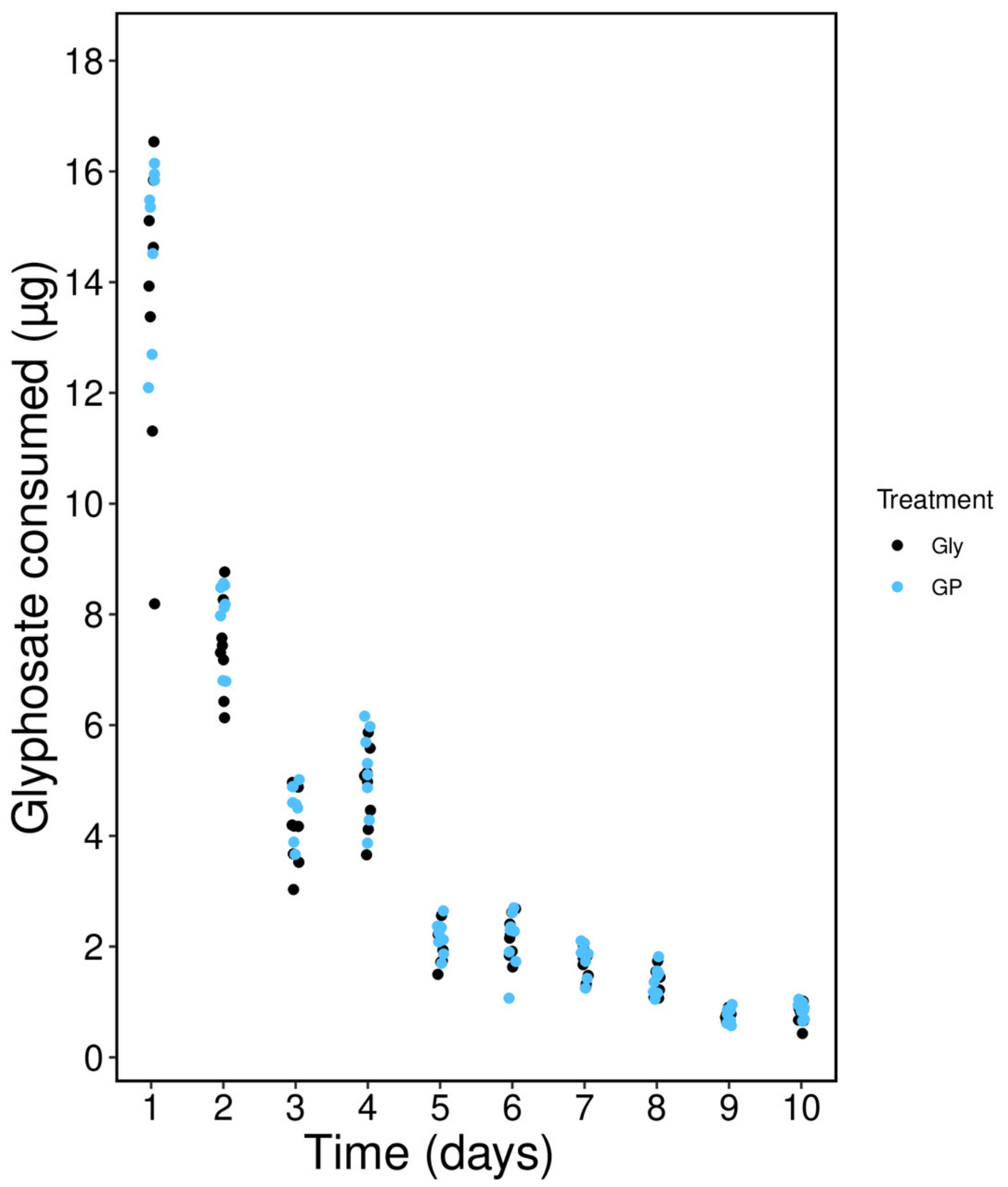


Figure 9

Microcolony Exposure - Chronic Exposure - Parasite Intensity

Figure 9. A boxplot with overlaid jittered data points showing the parasite intensity by treatment. 


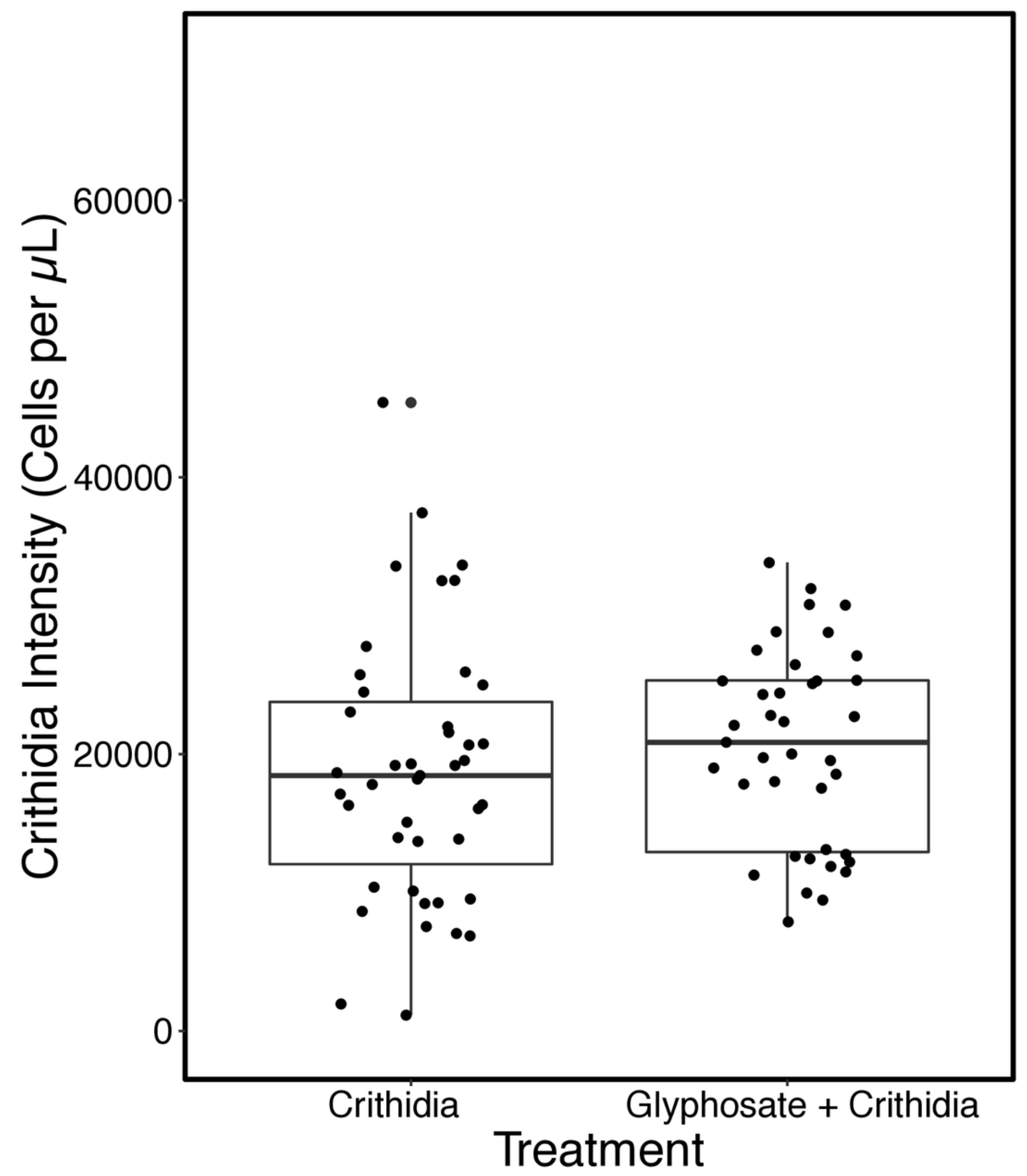

